PHYSICAL REVIEW D 81, 113005 (2010)

\title{
Quantum effects on Higgs-strahlung events at linear colliders within the general two-Higgs-doublet model
}

\author{
David López-Val* and Joan Solà ${ }^{\dagger}$ \\ High Energy Physics Group, Departament ECM, and Institut de Ciències del Cosmos, Universitat de Barcelona, Avinguda Diagonal \\ 647, E-08028 Barcelona, Catalonia, Spain \\ Nicolás Bernal \\ Centro de Física Teórica de Partículas (CFTP), Instituto Superior Técnico, Avenida Rovisco Pais, 1049-001 Lisboa, Portugal
}

(Received 31 March 2010; published 18 June 2010)

\begin{abstract}
The associated production of neutral Higgs bosons with the $Z^{0}$ gauge boson $\left(h^{0} Z^{0}, H^{0} Z^{0}\right)$ is investigated in the context of the future linear colliders, such as the ILC and CLIC, within the general two-Higgsdoublet model (2HDM). We compute the corresponding cross section for the processes $\mathrm{e}^{+} \mathrm{e}^{-} \rightarrow$ $\mathrm{Z}^{0} \mathrm{~h}^{0} / \mathrm{Z}^{0} \mathrm{H}^{0}$ at one-loop, including the full set of corrections at $\mathcal{O}\left(\alpha_{e w}^{3}\right)$ together with the leading $\mathcal{O}\left(\alpha_{e w}^{4}\right)$ terms, in full consistency with the available theoretical and phenomenological constraints. We find that the wave-function corrections to the external Higgs fields are the dominant source of the quantum effects, which turn out to be large and negative (e.g., $\delta \sigma / \sigma \sim-20 \% /-60 \%$ ) in all the $\sqrt{s}$ range, and located predominantly in the region around $\tan \beta \sim 1$ and moderate values of the parameter $\lambda_{5}$ (being $\left.\lambda_{5}<0\right)$. This behavior can be ultimately traced back to the enhancement potential of the triple Higgsboson self-couplings, a trademark feature of the 2HDM with no counterpart in the Higgs sector of the minimal supersymmetric standard model. Even under this substantial depletion of the one-loop-corrected signal (which is also highly distinctive with respect to the SM expectation for $\mathrm{e}^{+} \mathrm{e}^{-} \rightarrow \mathrm{Z}^{0} \mathrm{H}$ ), the predicted Higgs-strahlung rates comfortably reach a few tens of femtobarn, which means barely $\sim 10^{3}-10^{4}$ events per $500 \mathrm{fb}^{-1}$ of integrated luminosity. Because of their great complementarity, we argue that the combined analysis of the Higgs-strahlung events $\left(\mathrm{h}^{0} \mathrm{Z}^{0}, \mathrm{H}^{0} \mathrm{Z}^{0}\right)$ and the previously computed one-loop Higgs-pair production processes $\left(\mathrm{H}^{0} \mathrm{~A}^{0}\right)$ could be instrumental to probe the structure of the Higgs sector at future linac facilities.
\end{abstract}

DOI: 10.1103/PhysRevD.81.113005

PACS numbers: 12.15.-y, 12.15.Lk, 12.60.Fr

\section{INTRODUCTION}

More than 40 years since the seminal ideas were coined by a handful of theoretical pioneers [1], our present understanding of the electroweak symmetry-breaking (EWSB) phenomenon through the Higgs (Englert-Brout and Guralnik-Hagen-Kibble) mechanism is still rather incomplete and experimentally inconclusive. On the one hand, there is no compelling alternative to consistently embed the EWSB mechanism into the quantum field theoretical description of particle physics offered by the-in so many regards successful - standard model (SM) of the strong and electroweak interactions. On the other hand, we do not have a single phenomenological hint of the existence of elementary scalar fields, not to mention the fact that we do not understand how to make compatible the EWSB mechanism and its associated vacuum energy with fundamental problems of different scope, for example, in the domain of cosmology. Still, the possibility to describe the inner theoretical structure of the SM through the EWSB mechanism is so successful within the restricted particle physics do-

\footnotetext{
*dlopez@ecm.ub.es

†sola@ecm.ub.es

†nicolas.bernal@cftp.ist.utl.pt
}

main that it would not be wise, not even advisable, to cease our pursuit of the phenomenological implications of the Higgs mechanism paradigm until the limits of the current experimental possibilities.

It goes without saying that the quest for experimental evidences of the Higgs boson is a most preeminent milestone of the upcoming generation of collider facilities. Nonetheless, the future data might well reveal that the purportedly found Higgs boson actually belongs to a richer model structure, which might be grounded somewhere beyond the minimal conception of the SM, namely, of a single, spinless, fundamental constituent of matter. If so, a few more fundamental spinless constituents could appear. A particularly well-motivated extension is the two-Higgsdoublet model structure encompassed by the minimal supersymmetric standard model (MSSM) [2]. Here the physical Higgs-boson spectrum contains a couple of $\mathcal{C} \mathcal{P}$-even $\left(\mathrm{h}^{0}, \mathrm{H}^{0}\right)$, one $\mathcal{C} \mathcal{P}$-odd $\left(\mathrm{A}^{0}\right)$, and two charged Higgs bosons $\left(\mathrm{H}^{ \pm}\right)$, and the corresponding Higgs potential is highly constrained by the underlying supersymmetry (SUSY). One particular consequence of the latter is that SUSY invariance restricts all Higgs-boson self-interactions to be gaugelike. While this represents an economy from the point of view of the number of couplings in the potential, it is strongly imbalanced by the exceeding number of pa- 
rameters (mixings and masses) populating the other sectors of the MSSM. In addition, the pure gauge nature of the Higgs-boson self-couplings make them highly inconspicuous from the practical point of view, in the sense that they are unable to trigger any outstanding phenomenological signature. The core of the enhancement capabilities of the MSSM Lagrangian resides, instead, in the multifarious pattern of Yukawa couplings between the Higgs bosons and the quarks, as well as between quarks, squarks, and charginos/neutralinos. The rich interplay of opportunities that they give rise to has been extensively analyzed in the past within a plethora of processes; see, e.g., [3-7], and also [8-11] for reviews on the subject.

The two-Higgs $\mathrm{SU}_{L}(2)$-doublet structure of the Higgs sector in the MSSM constitutes a genuine prediction of the SUSY dynamics. Nonetheless, a more general architecture can appear in the form of a non-SUSY framework through the so-called general (unconstrained) two-Higgs-doublet model (2HDM). ${ }^{1}$ Although they share a common Higgsboson spectrum, the potentially most distinctive phenomenological features of both models are located in very different sectors. The most relevant observation here is that, in the absence of an underlying SUSY, the triple $(3 \mathrm{H})$ and quartic $(4 \mathrm{H})$ Higgs-boson self-interactions are no longer restrained to be purely gauge. This can have a tremendous impact, e.g., in the physics of the top quark in hadron colliders, as it was shown long ago in [12], and it can also trigger significant neutral flavor-changing interactions [13] that can perfectly compete with the SUSY ones $[14,15]$.

Much attention has been devoted to Higgs-boson production and decay in hadron colliders, extending from the ongoing Tevatron facility at Fermilab to the brand new LHC collider recently operating at CERN [8-17]. However, precision Higgs-boson physics will greatly benefit from the interplay [18] of the forthcoming generation of linear colliders (linac), such as the ILC and CLIC projects [19]. Here a variety of processes can provide new clues to Higgs-boson physics, e.g. the production of triple Higgsboson final states, both in the MSSM [20] and in the general 2HDM [21]; the double Higgs-strahlung channels $h h Z^{0}$ [22]; and the inclusive Higgs-pair production via gauge-boson fusion [20,23]. In the same vein, also the $\gamma \gamma$ mode of a linac has been explored [24], in particular, the loop-induced production of a single neutral Higgs boson [25] and of a Higgs-boson pair [26]. In all the above-mentioned cases, promising signatures were singled

\footnotetext{
${ }^{1}$ To be more precise, we refer to this model as "general" in the sense that we allow all possible operators leading to a renormalizable, gauge-invariant and $\mathcal{C P}$-conserving Higgs potential. As usually done in practice [11], we impose an additional (softly broken) $Z_{2}$ discrete symmetry $\Phi_{i} \rightarrow(-1)^{i} \Phi_{i}$, where $i=1,2$ denote each of the $\mathrm{SU}_{L}(2)$ Higgs doublets, as a sufficient condition to banish the tree-level flavor-changing neutral currents.
}

out and illustrate that, if effectively realized in nature, such hints of a generic 2HDM structure could hardly be missed in the superbly clean environment of a linac. What is more, they could not be confused as having a SUSY origin, because of the intrinsically different nature of the Higgs self-interaction sector. Besides, outstanding fingerprints of a generic 2HDM could also be stamped in the pattern of radiative corrections to Higgs production processes; for instance, quantum effects on the cross sections for twobody Higgs-boson final states,

$$
\mathrm{e}^{+} \mathrm{e}^{-} \rightarrow 2 h \quad\left(2 h \equiv \mathrm{h}^{0} \mathrm{~A}^{0} ; \mathrm{H}^{0} \mathrm{~A}^{0} ; \mathrm{H}^{+} \mathrm{H}^{-}\right),
$$

have been attentively investigated in the MSSM [27-30]. As for the general $2 \mathrm{HDM}$, the efforts were first concentrated on the production of charged Higgs pairs [31]. This program has been recently brought to completion from a full-fledged study of the quantum effects on the production cross sections in the neutral Higgs sector [32]. Alongside these processes we find the more traditional Higgsstrahlung events, in which a Higgs boson is produced in association with the $\mathrm{Z}^{0}$ :

$$
\mathrm{e}^{+} \mathrm{e}^{-} \rightarrow h \mathrm{Z}^{0}, \quad h=\mathrm{h}^{0}, \mathrm{H}^{0} .
$$

(Notice that the $\mathrm{A}^{0} \mathrm{Z}^{0}$ final state is forbidden by $\mathcal{C P}$ conservation.) Processes (2) are complementary to the (1) ones-cf. [33], and also [20,34] for a phenomenological analysis in the MSSM context. Let us also recall that the last available limit on the SM Higgs-boson mass, placed by LEP searches, comes precisely from investigating the "Bjorken process" [35], i.e. $\mathrm{e}^{+} \mathrm{e}^{-} \rightarrow \mathrm{HZ}^{0}$ followed by $\mathrm{Z}^{0} \rightarrow f \bar{f}$, with the result: $M_{H} \gtrsim 114.4 \mathrm{GeV}$ [36]. In this article, we aim at discussing the corresponding one-loop corrections to the generalized Bjorken processes (2) within the 2HDM. However, in contrast to the original SM case, where the radiative corrections are small, the quantum effects on the processes (2) can be large and are mainly driven by the $3 \mathrm{H}$ self-couplings. In fact, our main aim here is to identify the regions of the parameter space where this is so, and then quantify the impact of the potentially enhanced $3 \mathrm{H}$ self-couplings on the final cross sections. In this way, joining this study with that of Ref. [32] on the pairwise Higgs-boson production channels (1) in the 2HDM, a rather complete panorama of the genuine quantum effects associated to the basic neutral Higgs-boson production channels becomes available.

\section{HIGGS-STRAHLUNG EVENTS AT ONE-LOOP: THEORETICAL SETUP}

A good deal of attention has been devoted in the literature to multiple properties of the Higgs-strahlung process $\mathrm{e}^{+} \mathrm{e}^{-} \rightarrow \mathrm{HZ}^{0}$ in the SM, see e.g. [37] and references therein. Not surprisingly it was one of the gold-plated channels for Higgs-boson search at LEP. Concerning the MSSM, the Higgs-strahlung channels (2) have also been discussed extensively—cf. Refs. [28,29,38] and part two of 
the review [9] - and are currently under investigation also in the MSSM with $\mathcal{C P}$-violating phases [39] (the so-called complex MSSM [40]). The upshot of these analyses spotlights the following features: (i) the dominant source of corrections at one-loop originate from the Higgs-boson propagators, which can be reabsorbed into an effective (loop-corrected) mixing angle $\alpha_{\text {eff }}$ (equivalently, a more generic mixing matrix in the complex MSSM case); (ii) the corrections to the ZZh vertex are in general small, although they can reach the level of $10 \%$ for very low (or high) values of $\tan \beta$, precisely in the regions where the Higgs Yukawa couplings to heavy quarks become enhanced; (iii) the electromagnetic corrections to the initial state with virtual photonic corrections and initial-state radiation do not differ from the SM case, being in general large and positive (except near the production threshold). In the present article, our endeavor is to seek for the genuine phenomenological imprints associated to the generic (unconstrained) $2 \mathrm{HDM}$ dynamics, most particularly to the potentially enhanced $3 \mathrm{H}$ self-couplings.

Let us recall that the general 2HDM [11] is obtained by canonically extending the SM Higgs sector with a second $\mathrm{SU}_{L}(2)$ doublet with weak hypercharge $Y=+1$, so that it contains four complex scalar fields. The free parameters $\lambda_{i}$ in the general, $\mathcal{C} \mathcal{P}$-conserving, $2 \mathrm{HDM}$ potential can be finally expressed in terms of the masses of the physical Higgs particles $\left(M_{h^{0}}, M_{H^{0}}, M_{A^{0}}, M_{H^{ \pm}}\right), \tan \beta$ (the ratio of the two VEV's $\left\langle H_{i}^{0}\right\rangle$ giving masses to the uplike and downlike quarks), the mixing angle $\alpha$ between the two $\mathcal{C P}$-even states and, last but not least, the self-coupling $\lambda_{5}$, which cannot be absorbed in the previous quantities. Therefore we end up with a seven-free parameter set, to wit: $\left(M_{h^{0}}\right.$, $\left.M_{H^{0}}, M_{A^{0}}, M_{H^{ \pm}}, \sin \alpha, \tan \beta, \lambda_{5}\right)$. Furthermore, to ensure the absence of tree-level flavor-changing neutral currents, two main 2HDM scenarios arise: (1) type-I 2HDM, in which one Higgs doublet couples to all quarks, whereas the other doublet does not couple to them at all; (2) type-II 2HDM, where one doublet couples only to downlike quarks and the other doublet to uplike quarks. The MSSM Higgs sector is actually a type-II one, but of a very restricted sort (enforced by SUSY invariance) [11]. We refer the reader to Ref. [32] for a comprehensive account on the structure of the model and for notational details. In particular, Table II of that reference includes the full list of trilinear couplings within the general 2HDM that are relevant for the present calculation. Further constraints must be imposed to assess that the SM behavior is sufficiently well reproduced up to the energies explored so far. Most particularly, we take into account (i) the approximate SU(2) custodial symmetry, which can be reshuffled into the condition $\left|\delta \rho_{2 \mathrm{HDM}}\right| \leq 10^{-3}$ [41]; and (ii) the agreement with the low-energy radiative $B$-meson decay data (which demand $M_{H^{ \pm}} \geq 300 \mathrm{GeV}$ for $\tan \beta \geq 1$ [42] in the case of type-II 2HDM).

Additional requirements ensue from the theoretical consistency of the model, to wit (i) perturbativity [43]; (ii) unitarity [44]; and (iii) stability of the $2 \mathrm{HDM}$ vacuum [45]. Let us expand a bit more on the latter conditions, which turn out to play a crucial role in our analysis. As for the unitarity bounds, and following Ref. [44], the basic underlying strategy is to compute the $S$-matrix elements $S_{i j}$ for the possible $2 \rightarrow 2$ processes involving Higgs and Goldstone bosons in the 2HDM, and to subsequently restrain the corresponding eigenvalues $U_{i k} S_{k l} U_{l j}^{-1}=\alpha_{i} \delta_{i j}$ by the generic condition $\left|\alpha_{i}\right|<1 / 2 \forall i$. The latter requirement translates into a set of upper bounds on the quartic scalar couplings $\lambda_{i}, i=1-6$ - and hence on combinations of Higgs masses, trigonometric couplings, and, most significantly, the parameter $\lambda_{5}$. As far as vacuum stability restrictions are concerned [45], they may be written as follows:

$$
\begin{aligned}
& \lambda_{1}+\lambda_{3}>0 ; \quad \lambda_{2}+\lambda_{3}>0 ; \\
& 2 \sqrt{\left(\lambda_{1}+\lambda_{3}\right)\left(\lambda_{2}+\lambda_{3}\right)}+2 \lambda_{3}+\lambda_{4} \\
& +\min \left[0, \lambda_{5}-\lambda_{4}, \lambda_{6}-\lambda_{4}\right]>0 .
\end{aligned}
$$

More refined versions of these equations may be obtained from the renormalization group running of the quartic couplings $\lambda_{i}\left(\mu^{2}\right)$ at high energies. Nonetheless, for our current purposes we need not assume any particular UV completion of the 2HDM. This would be an unnecessary additional assumption at this stage of the phenomenological analysis of our processes. Therefore, we are not tied to any specific UV cutoff (which could be, in principle, as low as $\Lambda \sim 1-10 \mathrm{TeV}$ ). In this sense, we may just apply Eq. (3) with all $\lambda_{i}$ taken at the EW scale. This is after all the scale at which we fix all our input parameters and perform the phenomenological analysis (including the renormalization) of the processes under study. By the same token, the (cutoff dependent) triviality bounds are also circumvented. The latter kind of restrictions only impose tight upper limits on the Higgs-boson masses when a very large UV cutoff (e.g. the Planck scale or the GUT scale) is considered. Our choices of Higgs-boson mass spectra (cf. Table I) lie, in any case, in a sufficiently low mass range so as to conform with the typical mass requirements allowed by triviality [46] — even for large cutoff scales. More details on the constraints setup are provided in Ref. [32].

The dynamics of the Higgs-strahlung processes under consideration is driven at leading order by the tree-level interaction Lagrangians:

$$
\begin{aligned}
& \mathcal{L}_{\mathrm{Z}^{0} \mathrm{Z}^{0} \mathrm{~h}^{0}}=\frac{e \sin (\beta-\alpha) M_{Z}}{s_{W} c_{W}} g^{\mu \nu} \mathrm{Z}_{\mu}^{0} \mathrm{Z}_{\nu}^{0} \mathrm{~h}^{0}, \\
& \mathcal{L}_{\mathrm{Z}^{0} \mathrm{Z}^{0} \mathrm{H}^{0}}=\frac{e \cos (\beta-\alpha) M_{Z}}{s_{W} c_{W}} g^{\mu \nu} \mathrm{Z}_{\mu}^{0} \mathrm{Z}_{\nu}^{0} \mathrm{H}^{0},
\end{aligned}
$$

where $s_{W} \equiv \sin \theta_{W}$ and $c_{W} \equiv \cos \theta_{W}$ for the electroweak mixing angle $\theta_{W}$. Since $\mathcal{L}_{\mathrm{A}^{0} \mathrm{~h}^{0} \mathrm{Z}^{0}} \propto \cos (\beta-\alpha)$ and $\mathcal{L}_{\mathrm{A}^{0} \mathrm{H}^{0} \mathrm{Z}^{0}} \propto \sin (\beta-\alpha)$, it is clear that the processes (2) 
are complementary to the Higgs-pair production ones (1). Furthermore, as all these couplings (4) are generated by the gauged kinetic terms of the Higgs doublets [cf. Eq. (30) of Ref. [32] ], they are fully determined by the gauge symmetry and hence show no intrinsic difference in the 2HDM as compared to the MSSM. At the end of the day, this is the reason why a tree-level analysis of these events is most likely insufficient to disclose their true nature. Similarly, in the limit $\alpha=\beta-\pi / 2$, the $\mathrm{h}^{0} \mathrm{Z}^{0} \mathrm{Z}^{0}$ coupling coincides with the analogue coupling in the $\mathrm{SM}, \mathrm{HZ}^{0} \mathrm{Z}^{0}$. It is, therefore, the pattern of radiative corrections the characteristic signature associated to each one of the possible models; most particularly, it should help to disentangle SUSY versus non-SUSY extended Higgs physics scenarios.

The leading-order $\mathcal{O}\left(\alpha_{e w}\right)$ scattering amplitude follows from the $s$-channel $Z_{0}$-boson exchange and renders

$$
\begin{aligned}
\mathcal{M}^{(0)}\left(\mathrm{e}^{+} \mathrm{e}^{-} \rightarrow \mathrm{h}^{0} \mathrm{Z}^{0}\right)= & -\frac{e^{2} M_{Z} \sin (\beta-\alpha)}{s_{W} c_{W}\left(s-M_{Z}^{2}\right)} \times \bar{v}\left(p_{1}, \eta_{1}\right) \\
& \times \notin\left(k_{2}, \sigma_{2}\right)\left(g_{L} P_{L}+g_{R} P_{R}\right) \\
& \times u\left(p_{2}, \eta_{2}\right) .
\end{aligned}
$$

Here $p_{1,2}$ and $\eta_{1,2}$ refer to the 4-momenta and helicities of the electron and positron, and $\epsilon\left(k_{2}, \sigma_{2}\right)$ is the polarization 4 -vector of the $Z$ gauge boson with 4-momentum $k_{2}$ and helicity $\sigma_{2}$. We have introduced also the left- and righthanded weak couplings of the $Z_{0}$ boson to the electron, $g_{L}=\left(-1 / 2+s_{W}^{2}\right) / c_{W} s_{W}, g_{R}=s_{W} / c_{W}$, and the left and right-handed projectors $P_{L, R}=(1 / 2)\left(1 \mp \gamma_{5}\right)$. Let us notice that we do not include the finite $Z^{0}$-width corrections, since they are completely negligible for the center-of-mass energies that we consider here. Finally, the total cross section $\sigma\left(\mathrm{e}^{+} \mathrm{e}^{-} \rightarrow \mathrm{h}^{0} \mathrm{Z}^{0}\right)$ at the tree level is obtained after squaring the matrix element (5), performing an averaged sum over the polarizations of the colliding $\mathrm{e}^{+} \mathrm{e}^{-}$beams and the outflowing $Z^{0}$ boson, and integrating over the scattering angle.

The calculation of $\sigma\left(\mathrm{e}^{+} \mathrm{e}^{-} \rightarrow \mathrm{h}^{0} \mathrm{Z}^{0}\right)$ at one-loop is certainly much more cumbersome. To start with, it is UV divergent and it thus requires of a careful renormalization procedure in order to render finite results. We adopt here the conventional on-shell scheme in the Feynman gauge [47]. In the MSSM, these cross-section calculations can be mostly carried out in an automatized fashion through standard algebraic packages which allow a rapid and efficient analysis of the electroweak precision observables [48]. Several public codes are available, see e.g. [49-52]. However, in our case the calculation is nonsupersymmetric and we must deal with the renormalization of the Higgs sector in the class of generic 2HDM models. A detailed description of the renormalization procedure for the 2HDM Higgs sector in the on-shell scheme has been presented in [32] and we refer the reader to this reference for all the necessary details. We will make constant use of the framework described in this reference and sometimes we will refer to particular formulas of it.

With this renormalization setup in mind, the various contributions to the scattering amplitude of $\mathrm{e}^{+} \mathrm{e}^{-} \rightarrow \mathrm{h}^{0} \mathrm{Z}^{0}$ at one-loop (Figs. 1 and 2) can be classified in a meaningful way. First of all, let us note that all of the one-loop diagrams are of course of order $\mathcal{O}\left(\alpha_{e w}^{2}\right)$. However, some of them include, in addition, enhancement factors sourced by the trilinear Higgs-boson couplings $\lambda_{3 H}$, see Table II of [32]. In such cases, we shall include these factors when assessing the order of magnitude of the diagram. We are now ready for sorting out the one-loop contributions in different categories:
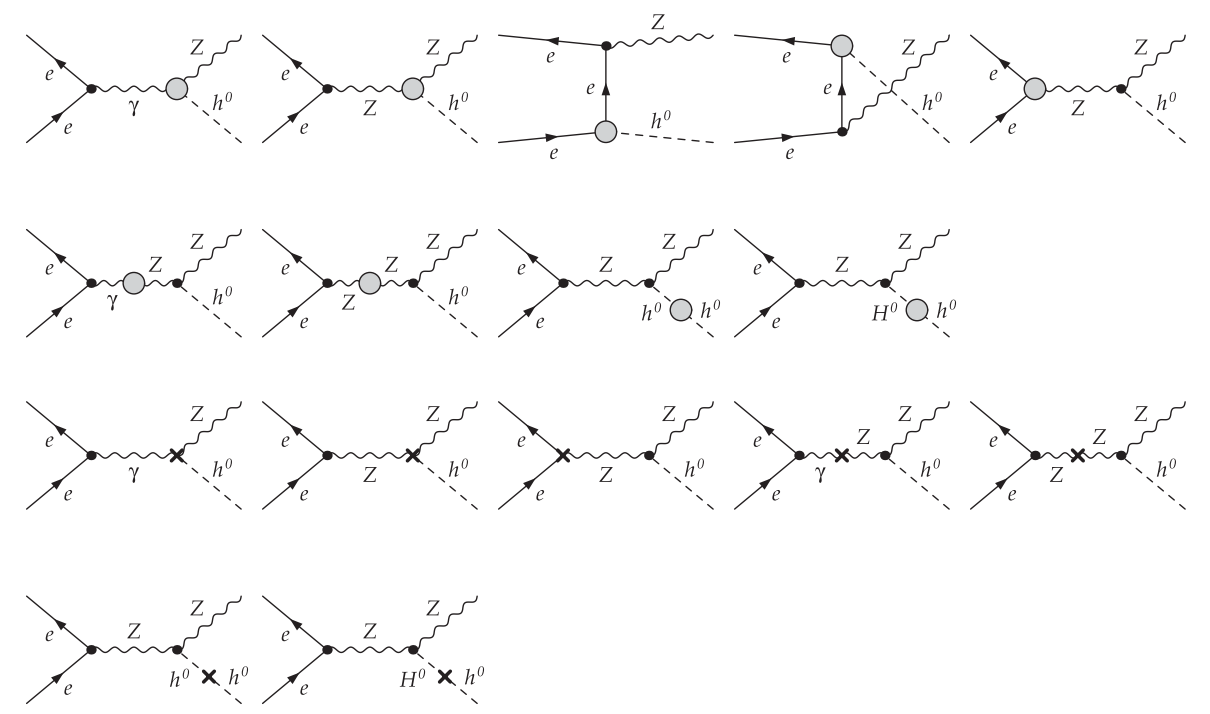

FIG. 1. Set of Feynman diagrams contributing to $\mathrm{e}^{+} \mathrm{e}^{-} \rightarrow \mathrm{h}^{0} \mathrm{Z}^{0}$ at one-loop level within the 2HDM. The shaded blobs stand for all possible loops with virtual particles. An equivalent collection of diagrams accounts for the complementary process $\mathrm{e}^{+} \mathrm{e}^{-} \rightarrow \mathrm{H}^{0} \mathrm{Z}^{0}$. The last couple of rows displays the complete set of one-loop counterterm diagrams. 

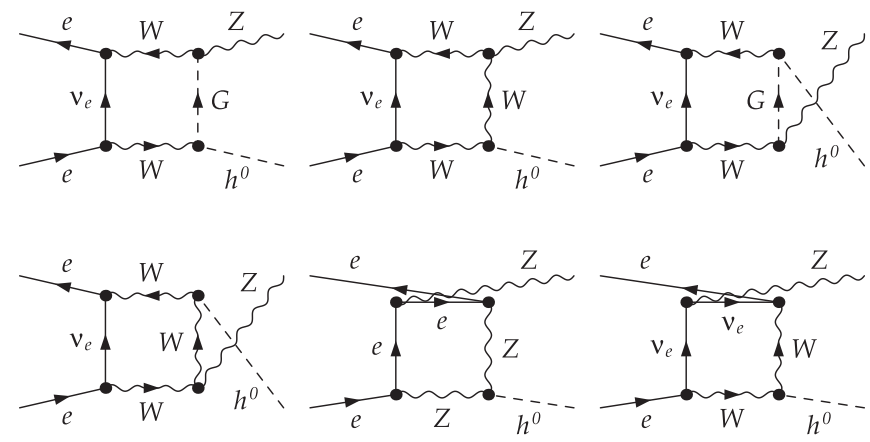

FIG. 2. Set of Feynman diagrams contributing to $\mathrm{e}^{+} \mathrm{e}^{-} \rightarrow$ $\mathrm{h}^{0} \mathrm{Z}^{0}$ at one-loop level within the $2 \mathrm{HDM}$. These diagrams describe the box-type quantum corrections. An equivalent collection of diagrams accounts for the complementary process $\mathrm{e}^{+} \mathrm{e}^{-} \rightarrow \mathrm{H}^{0} \mathrm{Z}^{0}$.

(i) Self-energy corrections to the $Z^{0}$ and $\gamma-Z^{0}$ mixing propagators, all of them of order $\mathcal{O}\left(\alpha_{e w}^{2}\right)$ with no enhancement factors at this order.

(ii) Vertex corrections to the $\mathrm{e}^{+} \mathrm{e}^{-} \mathrm{Z}^{0}$ interaction, which are also of order $\mathcal{O}\left(\alpha_{e w}^{2}\right)$. It should be noted that we do not include the virtual photonic $\mathcal{O}\left(\alpha_{e m} \alpha_{e w}\right)$ effects nor the real bremsstrahlung emission off the $\mathrm{e}^{+} / \mathrm{e}^{-}$legs. These pure QED corrections and the weak ones factorize into two subsets which are separately UV finite and gauge invariant. Moreover, these photonic contributions are fully insensitive, at the order under consideration, to the relevant $3 \mathrm{H}$ self-couplings on which we focus. For these kinds of processes involving electrically neutral Higgs bosons in the final state, the one-loop QED effects are confined to the initial $\mathrm{e}^{+} / \mathrm{e}^{-}$vertex. In practice, the net outcome of the accompanying initial-state radiation is to lower the effective center-of-mass energy available for the annihilation process. All in all, these effects are unessential at this stage to test the presence of the new dynamical features triggered by the $2 \mathrm{HDM}$ in the Higgsstrahlung events under analysis.

(iii) Vertex corrections to the $\mathrm{e}^{+} \mathrm{e}^{-} \mathrm{H}$ interaction. Since we have explicitly set $m_{e}=0$ throughout our calculation, the $\mathrm{e}^{+} \mathrm{e}^{-} \mathrm{H}$ tree-level Yukawa coupling is absent and the corresponding one-loop diagrams automatically render a UV-finite contribution which is, in any case, very small.

(iv) The loop-induced $\gamma \mathrm{Z}^{0} \mathrm{~h}^{0}$ interaction. This one is order $\mathcal{O}\left(e \alpha_{e w} \lambda_{3 H}\right)$ and therefore includes an enhancement factor $\lambda_{3 H}$. Because of the $\gamma-\mathrm{Z}^{0}$ mixing at one-loop, the following counterterm is needed so as to render a UV-finite vertex:

$$
\delta \mathcal{L}_{\mathrm{h}^{0} Z^{0} \gamma}=\frac{e \sin (\beta-\alpha) M_{Z}}{2 s_{W} c_{W}} \delta Z_{\gamma Z^{0}} g^{\mu \nu} Z_{\mu}^{0} A_{\nu} \mathrm{h}^{0}
$$

and similarly for the counterterm associated to the effective $\mathcal{L}_{\mathrm{H}^{0} Z^{0} \gamma}$ interaction:

$$
\delta \mathcal{L}_{\mathrm{H}^{0} Z^{0} \gamma}=\frac{e \cos (\beta-\alpha) M_{Z}}{2 s_{W} c_{W}} \delta Z_{\gamma Z^{0}} g^{\mu \nu} Z_{\mu}^{0} A_{\nu} \mathrm{H}^{0},
$$

(v) The vertex correction for $h Z^{0} Z^{0}$ encompasses different contributions of order $\mathcal{O}\left(\alpha_{e w}^{2}, \alpha_{e w} e \lambda_{3 H}\right)$ (see the sample diagrams in Fig. 3). Most important, there are, in addition, corrections of $\mathcal{O}\left(\alpha_{e w} \lambda_{3 H}^{2}\right)$ (in fact, the dominant ones) which are characterized by the highly conspicuous enhancement factor squared $\lambda_{3 H}^{2}$. The latter originates from the associated vertex counterterm, most particularly from the Higgs field renormalization constant $Z_{\mathrm{h}^{0}}^{1 / 2}=1+(1 / 2) \delta Z_{\mathrm{h}^{0}}$. As we shall discuss in more detail below, $\delta Z_{\mathrm{h}^{0}}$ is sensitive to the scalar-scalar self-energy and hence it involves products of two triple Higgs self-couplings (see Fig. 4). Of course, an equivalent discussion holds for the complementary channel, $\mathrm{H}^{0} \mathrm{Z}^{0}$.

The full form of the associated one-loop vertex counterterms ensues from the usual splitting of bare fields and parameters into the renormalized ones and associated counterterms, to wit:

$$
\mathcal{L}\left(g_{0}=g+\delta g, \phi_{0}=Z_{i}^{1 / 2} \phi\right) \rightarrow \mathcal{L}(g, \phi)+\delta \mathcal{L} .
$$

For the $h^{0} Z^{0} Z^{0}$ and $H^{0} Z^{0} Z^{0}$ case, one gets, respectively,

$$
\begin{aligned}
\delta \mathcal{L}_{\mathrm{h}^{0} Z^{0} Z^{0}}= & \frac{e \sin (\beta-\alpha) M_{Z}}{s_{W} c_{W}}\left[\frac{s_{W}^{2}-c_{W}^{2}}{c_{W}^{2}} \frac{\delta s_{W}}{s_{W}}+\sin \beta\right. \\
& \times \cos \beta \cot (\beta-\alpha) \frac{\delta \tan \beta}{\tan \beta}+\frac{\delta e}{e}+\frac{\delta M_{Z}^{2}}{2 M_{Z}^{2}} \\
& +\frac{1}{2} \delta Z_{\mathrm{h}^{0}}+\frac{1}{2} \delta Z_{\mathrm{Z}^{0}}+\frac{1}{2} \cot (\beta-\alpha) \\
& \left.\times \delta Z_{\mathrm{H}^{0} \mathrm{~h}^{0}}\right] g^{\mu \nu} \mathrm{Z}_{\mu}^{0} \mathrm{Z}_{\nu}^{0} \mathrm{~h}^{0},
\end{aligned}
$$
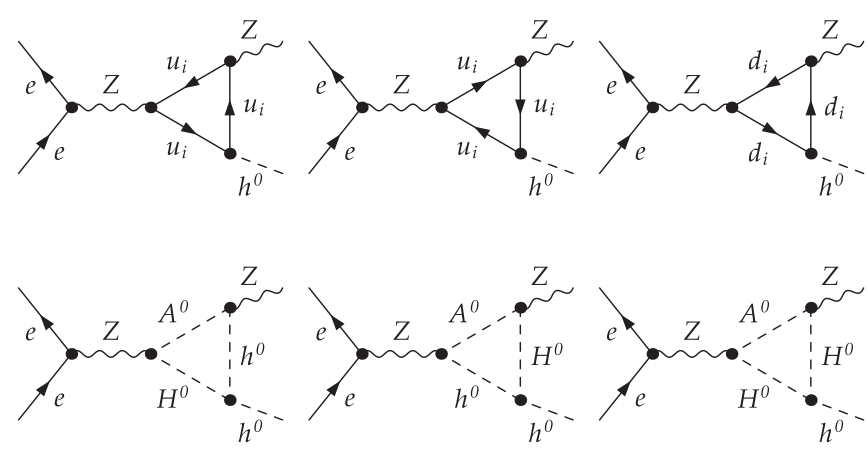

FIG. 3. Sample of Feynman diagrams describing the one-loop corrections to the $\mathrm{h}^{0} \mathrm{Z}^{0} \mathrm{Z}^{0}$ interaction for the process $\mathrm{e}^{+} \mathrm{e}^{-} \rightarrow$ $\mathrm{h}^{0} \mathrm{Z}^{0}$ within the $2 \mathrm{HDM}$, including quark-mediated (top line) and Higgs-boson-mediated quantum corrections (bottom line). 


$$
\begin{aligned}
\delta \mathcal{L}_{\mathrm{H}^{0} \mathrm{Z}^{0} \mathrm{Z}^{0}}= & \frac{e \cos (\beta-\alpha) M_{Z}}{s_{W} c_{W}}\left[\frac{s_{W}^{2}-c_{W}^{2}}{c_{W}^{2}} \frac{\delta s_{W}}{s_{W}}-\sin \beta\right. \\
& \times \cos \beta \tan (\beta-\alpha) \frac{\delta \tan \beta}{\tan \beta}+\frac{\delta e}{e}+\frac{\delta M_{Z}^{2}}{2 M_{Z}^{2}} \\
& +\frac{1}{2} \delta Z_{\mathrm{H}^{0}}+\frac{1}{2} \delta Z_{\mathrm{Z}^{0}}+\frac{1}{2} \tan (\beta-\alpha) \\
& \left.\times \delta Z_{\mathrm{H}^{0} \mathrm{~h}^{0}}\right] g^{\mu \nu} \mathrm{Z}_{\mu}^{0} \mathrm{Z}_{\nu}^{0} \mathrm{H}_{0},
\end{aligned}
$$

where we can spot in the structure of these formulas the presence of the above-mentioned Higgs field renormalization counterterms $\delta Z_{\mathrm{h}^{0}}$ and $\delta Z_{\mathrm{H}^{0}}$. The term $\delta Z_{\mathrm{h}^{0} \mathrm{H}^{0}}$ accounts for the wave-function (WF) mixing $\mathrm{h}^{0} \leftrightarrow \mathrm{H}^{0}$ emerging from the $\mathrm{Z}^{0} \mathrm{Z}^{0} \mathrm{~h}^{0}$ and $\mathrm{Z}^{0} \mathrm{Z}^{0} \mathrm{H}^{0}$ vertices.

(vi) The finite WF correction to the external Higgs fields $\mathrm{h}^{0}$ or $\mathrm{H}^{0}$ (Fig. 4). These are related to the fact that we have chosen the residue of the $\mathrm{A}^{0}$ propagator at its pole to be one, and therefore there is no more freedom to make the same choice for the other Higgs bosons. This entails a finite WF renormalization correction; see Ref. [32] for details. These finite renormalization effects are of utmost importance in this case, as they trigger leading contributions of order $\mathcal{O}\left(\alpha_{e w} \lambda_{3 H}^{2}\right)$. They actually drive the very bulk of the quantum effects (see below for a more detailed discussion).

(vii) Finally, the box-type $\mathcal{O}\left(\alpha_{e w}^{2}\right)$ diagrams (Fig. 2), whose contribution is non-negligible, in particular, at large center-of-mass energies-since they are not suppressed as $1 / s$.

The dominance of the WF corrections is a very characteristic feature of the processes under analysis. It originates from a nontrivial cancellation between the Higgs-boson field counterterms, which appear in two different pieces of the overall one-loop amplitude, though with opposite signs. Let us further elaborate on this important point. Without loss of generality, we concentrate on the $h^{0} Z^{0}$ channel. The complete set of contributions to the scattering amplitude up to the one-loop level may be split in the following manner:

$$
\begin{aligned}
\mathcal{M}_{\mathrm{e}^{+} \mathrm{e}^{-} \rightarrow \mathrm{h}^{0} Z^{0}}^{(0+1)} & \mathcal{M}_{\mathrm{e}^{+} \mathrm{e}^{-} \rightarrow \mathrm{h}^{0} Z^{0}}^{(0)}+\mathcal{M}_{\mathrm{e}^{+} \mathrm{e}^{-} \rightarrow \mathrm{h}^{0} Z^{0}}^{(1)} \\
& +\delta \mathcal{M}_{\mathrm{e}^{+} \mathrm{e}^{-} \rightarrow \mathrm{h}^{0} \mathrm{Z}^{0}}^{(1)}+\mathcal{M}_{\mathrm{e}^{+} \mathrm{e}^{-} \rightarrow \mathrm{h}^{0} Z^{0},}^{\mathrm{WF}}
\end{aligned}
$$

wherein the finite WF corrections to the external Higgs field are introduced as

$$
\begin{aligned}
\mathcal{M}_{\mathrm{e}^{+} \mathrm{e}^{-} \rightarrow \mathrm{h}^{0} Z^{0}}^{\mathrm{WF}} & =\left(\sqrt{\hat{Z}_{\mathrm{h}^{0}}}-1\right) \mathcal{M}_{\mathrm{e}^{+} \mathrm{e}^{-} \rightarrow \mathrm{h}^{0} Z^{0}}^{(0)} \\
& =-\frac{1}{2} \underbrace{\operatorname{Re} \hat{\Sigma}_{\mathrm{h}^{0}}^{\prime}\left(M_{\mathrm{h}^{0}}^{2}\right)}_{\mathcal{O}\left(\lambda_{3 H}^{2}\right)} \mathcal{M}_{\mathrm{e}^{+} \mathrm{e}^{-} \rightarrow \mathrm{h}^{0} Z^{0}}^{(0)}+\mathcal{O}\left(\alpha_{e w}^{3}\right) .
\end{aligned}
$$

At this point we are making explicit use of the on-shell renormalization conditions defined in [32], in particular $\operatorname{Re} \hat{\Sigma}_{\mathrm{h}^{0} \mathrm{H}^{0}}\left(M_{\mathrm{h}^{0}}^{2}\right)=0$. Moreover, we only retain those contributions that are leading order in the triple Higgs selfcouplings. Let us notice that the counterterm piece $\delta \mathcal{M}^{(1)}$ is sensitive to $\mathcal{O}\left(\lambda_{3 H}^{2}\right)$ effects through the WF renormalization of the Higgs fields. More specifically, from the explicit expression of the $\mathrm{h}^{0}$-field counterterm as a linear combination of the two-Higgs-doublet counterterms (see Sec. IV of Ref. [32] for details)

$$
\begin{aligned}
\delta Z_{\mathrm{h}^{0}} & =\sin ^{2} \alpha \delta Z_{\Phi_{1}}+\cos ^{2} \alpha \delta Z_{\Phi_{2}} \\
& =-\operatorname{Re} \Sigma_{\mathrm{A}^{0}}^{\prime}\left(M_{\mathrm{A}^{0}}^{2}\right)+\cdots,
\end{aligned}
$$

we may single out the following $\mathcal{O}\left(\lambda_{3 H}^{2}\right)$ contribution from Eq. (9):

$$
\begin{aligned}
\delta \mathcal{L}_{\mathrm{h}^{0} Z^{0} Z^{0}} & \rightarrow \frac{e \sin (\beta-\alpha)}{2 s_{W} c_{W}} \delta Z_{\mathrm{h}^{0}}+\cdots \\
& =-\frac{e \sin (\beta-\alpha)}{2 s_{W} c_{W}} \underbrace{\operatorname{Re} \Sigma_{\mathrm{A}^{0}}^{\prime}\left(M_{\mathrm{A}^{0}}^{2}\right)}_{\mathcal{O}\left(\lambda_{3 H}^{2}\right)}+\cdots .
\end{aligned}
$$

But, as warned, the finite WF renormalization factor of the $\mathrm{h}^{0}$ field in (12) is sensitive to $\mathcal{O}\left(\lambda_{3 H}^{2}\right)$ terms too:

$$
\begin{aligned}
\operatorname{Re} \hat{\Sigma}_{\mathrm{h}^{0}}^{\prime}\left(M_{\mathrm{h}^{0}}^{2}\right) & =\underbrace{\operatorname{Re} \Sigma_{\mathrm{h}^{0}}^{\prime}\left(M_{\mathrm{h}^{0}}^{2}\right)+\delta Z_{\mathrm{h}^{0}}}_{\mathcal{O}\left(\lambda_{3 H}^{2}\right)} \\
& =\underbrace{\operatorname{Re} \Sigma_{\mathrm{h}^{0}}^{\prime}\left(M^{2}\right)-\operatorname{Re} \Sigma_{\mathrm{A}^{0}}^{\prime}\left(M_{\mathrm{A}^{0}}^{2}\right)}_{\mathrm{h}^{0}}+\cdots,
\end{aligned}
$$

where the dots stand for those terms with dependencies other than $\mathcal{O}\left(\lambda_{3 H}^{2}\right)$. Notice, therefore, that part of the $\mathcal{O}\left(\lambda_{3 H}^{2}\right)$ dependence cancels out between the counterterm diagram associated to the $h^{0} Z^{0} Z^{0}$ vertex — cf. Eq. (14) and the finite $\mathrm{h}^{0}$ WF-factor-cf. Eqs. (12) and (15). Specifically, it is the piece $\Re e \Sigma_{\mathrm{A}^{0}}^{\prime}\left(M_{\mathrm{A}^{0}}^{2}\right)$ that exactly cancels between the two terms. The remaining $\mathcal{O}\left(\lambda_{3 H}^{2}\right)$ contributions come from $\Re e \Sigma_{\mathrm{h}^{0}}^{\prime}\left(M_{\mathrm{h}^{0}}^{2}\right)$ and are generated by the Feynman diagrams displayed in Fig. 4. When sufficiently enhanced, these pieces account for the bulk of the one-loop quantum corrections. A typical diagram in the class of twopoint functions provides the following contribution: ${ }^{2}$

\footnotetext{
${ }^{2}$ Notice that we employ the convention that $i \Sigma$ equals the Higgs-boson self-energy diagram, such that $\Sigma$ does not contain the global imaginary part emerging from the loop integral. This is why we have multiplied the loop integral in (16) by $-i$ before taking the real part.
} 

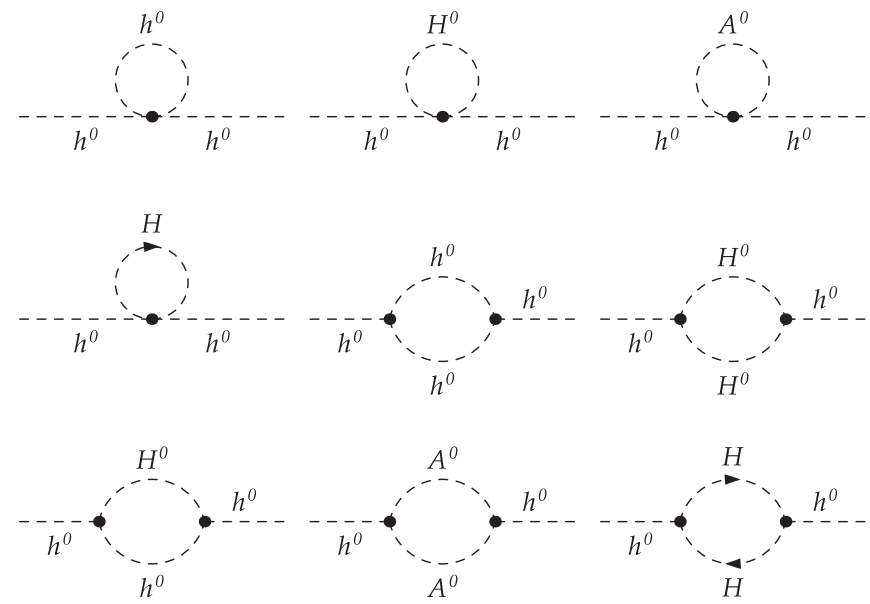

FIG. 4. Subset of Feynman diagrams driving the dominant contribution to the $\mathrm{h}^{0}$ boson self-energy. Notice that the twopoint functions are sensitive to two triple Higgs-boson selfcouplings. The tadpole diagrams, however, which depend on quartic couplings, do not contribute to the WF renormalization. An equivalent collection of diagrams contributes to the corresponding $\mathrm{H}^{0}$ self-energy.

$$
\begin{aligned}
\mathcal{M}_{\mathrm{e}^{+} \mathrm{e}^{-} \rightarrow \mathrm{h}^{0} \mathrm{Z}^{0}}^{(1)} & -\frac{1}{2} \operatorname{Re}_{\mathrm{h}^{0}}^{\prime}\left(M_{\mathrm{h}^{0}}^{2}\right) \mathcal{M}_{\mathrm{e}^{+} \mathrm{e}^{-} \rightarrow \mathrm{h}^{0} \mathrm{Z}^{0}}^{(0)} \\
\simeq & -\frac{1}{2} \mathcal{M}_{\mathrm{e}^{+} \mathrm{e}^{-} \rightarrow \mathrm{h}^{0} \mathrm{Z}^{0}}^{(0)}\left|\lambda_{3 H}\right|^{2} \operatorname{Re} \frac{d^{2}}{d p^{2}}(-i) \\
& \times \int \frac{d^{D} q}{(2 \pi)^{D}} \frac{\mu^{4-D}}{\left(q^{2}-M^{2}\right)\left[(q+p)^{2}-M^{2}\right]} \\
\sim & -\frac{1}{2} \frac{\left|\lambda_{3 H}\right|^{2}}{16 \pi^{2}} \mathcal{M}_{\mathrm{e}^{+} \mathrm{e}^{-} \rightarrow \mathrm{h}^{0} \mathrm{Z}^{0}}^{(0)} \operatorname{Re} B_{0}^{\prime}\left(M_{\mathrm{h}^{0}}^{2}, M^{2}, M^{2}\right),
\end{aligned}
$$

wherein the scalar two-point function is defined as in Ref. [49]:

$$
\begin{aligned}
& \int \frac{d^{D} q}{(2 \pi)^{D}} \frac{\mu^{4-D}}{\left(q^{2}-m_{1}^{2}\right)\left[(p+q)^{2}-m_{2}^{2}\right]} \\
& \equiv \frac{i}{16 \pi^{2}} B_{0}\left(p^{2}, m_{1}^{2}, m_{2}^{2}\right),
\end{aligned}
$$

$\mu$ denoting the 't Hooft mass unit. In the expression (16), $M$ denotes the typical mass scale(s) which appear in these one-loop two-point functions. Owing to the derivative with respect to the external momentum, the one-point functions do not contribute to the WF renormalization, and hence the quartic Higgs-boson self-couplings are not involved in this calculation. This means that the first line of diagrams in Fig. 4 does not actually contribute. We emphasize that the overall sign for this expression depends on the sign of such a two-point function. Notice that $\operatorname{Re} B_{0}^{\prime}\left(p^{2}, M^{2}, M^{2}\right)>0$ for $p^{2}<4 M^{2}$, which is the case we wish to focus on for the $\mathrm{h}^{0} \mathrm{Z}^{0}$ channel, and also for $\mathrm{H}^{0} \mathrm{Z}^{0}$ (as long as the resonant decay $\mathrm{H}^{0} \rightarrow \mathrm{h}^{0} \mathrm{~h}^{0}$ is forbidden by kinematics). Therefore, in most of the scenarios of interest, such finite WF correc- tions carry an overall minus sign. Being proportional to $\lambda_{3 H}^{2}$, they become the leading quantum effects in the region where the trilinear couplings are enhanced, and as a result they generate a characteristic pattern of quantum effects in which a systematic suppression of the tree-level cross section is predicted.

The presence of the large negative corrections induced by the Higgs-boson self-energies brings forward a characteristic signature for the production cross sections of the Higgs-strahlung processes (2). This feature is in marked contradistinction to the situation with the Higgs-boson pair production mechanisms (1), where the corresponding corrections are just opposite in sign, i.e. large and positive, see [32]. We shall further comment on these interesting and correlated features in the next section. From the general structure of Eq. (16), one may anticipate the typical (maximum) size of the quantum effects on the Higgs-strahlung processes as follows:

$$
\begin{aligned}
\delta_{r} & =\frac{\sigma^{(0+1)}-\sigma^{(0)}}{\sigma^{(0)}}=\frac{\left\langle 2 \mathcal{M}^{(0)} \mathcal{M}^{(1)}\right\rangle}{\left\langle\left|\mathcal{M}^{(0)}\right|^{2}\right\rangle} \\
& \simeq-\frac{\left|\lambda_{3 H}\right|^{2}}{16 \pi^{2} M^{2}} f\left(M_{\mathrm{h}^{0}}^{2}, M^{2}, M^{2}\right)
\end{aligned}
$$

$f$ being a dimensionless form factor (basically accounting for the behavior of the $B_{0}^{\prime}$ function). The notation $\langle\cdots\rangle$ stands for the various operations of averaging and integration of the squared matrix elements. Taking into account that unitarity limits let the trilinear couplings reach values as large as $\left(\lambda_{3 H}\right) / M_{W} \simeq \frac{\left|\lambda_{5}\right|}{e} \simeq 30$, and assuming $M \sim$ $200 \mathrm{GeV}$ and $f \sim \mathcal{O}(1)$, the above estimate typically predicts a strong depletion of the tree-level signal by $\delta_{r} \simeq$ $-90 \%$. This prediction falls in the right ballpark of the exact numerical results that will be reported in the next section, which point to a maximum depletion of $\delta_{r} \sim$ $-60 \%$.

Before closing this section, let us recall that the counterterm amplitude $\delta \mathcal{M}_{\mathrm{e}^{+} \mathrm{e}^{-} \rightarrow \mathrm{h}^{0} \mathrm{Z}^{0}}$ derives from a number of renormalization conditions that determine the renormalized coupling constants and fields in a given renormalization framework. As we have said, in our calculation the renormalization is performed in the conventional on-shell scheme in the Feynman gauge, appropriately extended to include the 2HDM Higgs sector. For the latter, we need, in particular, a renormalization condition for the parameter $\tan \beta=v_{2} / v_{1}$. We adopt the following [53]:

$$
\frac{\delta v_{1}}{v_{1}}=\frac{\delta v_{2}}{v_{2}} .
$$

This condition ensures that the ratio $v_{2} / v_{1}$ is always expressed in terms of the true vacua after the renormalization of the Higgs potential. The corresponding counterterm resulting from $\tan \beta \rightarrow \tan \beta+\delta \tan \beta$ can then be computed explicitly: 


$$
\frac{\delta \tan \beta}{\tan \beta}=\frac{1}{M_{Z} \sin 2 \beta} \Re e \Sigma_{\mathrm{A}^{0} Z^{0}}\left(M_{\mathrm{A}^{0}}^{2}\right) .
$$

This counterterm is involved in Eqs. (9) and (10), and it also determines the WF mixing term $\delta Z_{\mathrm{h}^{0} \mathrm{H}^{0}}$ that appears in these equations, as follows: $\delta Z_{\mathrm{h}^{0} \mathrm{H}^{0}}=\sin 2 \alpha(\delta \tan \beta / \tan \beta)$. We refer the reader once more to the exhaustive presentation of Ref. [32] for the renormalization details.

\section{NUMERICAL ANALYSIS}

In this section, we present the numerical analysis of the one-loop computation of the Higgs-strahlung processes (2) within the 2HDM. We shall be concerned basically with the following two quantities: (i) the predicted cross section at the Born level $\sigma^{(0)}$ and at one-loop $\sigma^{(0+1)}$; and (ii) the relative size of the one-loop quantum corrections:

$$
\delta_{r}=\frac{\sigma^{(0+1)}-\sigma^{(0)}}{\sigma^{(0)}} .
$$

We have carried out our analysis with the help of the standard algebraic and numerical tools FEYNARTS, FORMCALC and LOOPTOOLS [49].

The different Higgs-boson mass sets that shall be used hereafter are quoted in Table I. Let us highlight that, due to their mass splittings (and also to the fact that $M_{\mathrm{h}^{0}} \geq$ $140 \mathrm{GeV}$ ), sets C and D can only be realized in a general (non-SUSY) 2HDM framework. Furthermore, in view of the value of the charged Higgs mass, sets A-C are only suitable for type-I 2HDM, whereas set D is valid for either type-I and type-II 2HDM's.

Apart from reflecting a variety of possible situations in the 2HDM parameter space, some of these sets can be mimicked by the Higgs-boson mass spectrum in supersymmetric theories. For instance, sets A and B can be ascribed to characteristic benchmark scenarios of Higgsboson mass spectra within the MSSM; in particular, set B lies in the class of the so-called maximal mixing scenarios [54], for which $M_{\mathrm{h}^{0}}$ takes the highest possible values within the MSSM. The numerical mass values for the MSSM-like sets have been obtained with the aid of the program FEYNHIGGS by taking the full set of EW corrections at one-loop [50].

TABLE I. Choices of Higgs masses (in $\mathrm{GeV}$ ) that are used throughout the calculation. Because of the values of the $M_{\mathrm{H}^{ \pm}}$ mass, sets A-C would only be suitable for type-I 2HDM, while set D could account for both type-I and type-II models. We also notice that sets A, B have been devised in order to mimic the characteristic mass splittings of the MSSM Higgs sector.

\begin{tabular}{lcccc}
\hline \hline & $M_{\mathrm{h}^{0}}[\mathrm{GeV}]$ & $M_{\mathrm{H}^{0}}[\mathrm{GeV}]$ & $M_{\mathrm{A}^{0}}[\mathrm{GeV}]$ & $M_{\mathrm{H}^{ \pm}}[\mathrm{GeV}]$ \\
\hline Set A & 115 & 220 & 220 & 235 \\
Set B & 130 & 160 & 150 & 170 \\
Set C & 140 & 150 & 200 & 200 \\
Set D & 150 & 200 & 260 & 300 \\
\hline \hline
\end{tabular}

We remark that the more massive the Higgs bosons are, the stronger the constraints that unitarity imposes over $\left|\lambda_{5}\right|$. The maximum (negative) values are roughly attained for $\lambda_{5} \simeq-9($ set $\mathrm{A}) ; \lambda_{5} \simeq-10$ (sets B and C); and $\lambda_{5} \simeq-8$ (set D).

Before coming to grips with the analysis of the Higgsstrahlung events in the general $2 \mathrm{HDM}$, it is interesting to briefly reconsider the analogue process in the simpler framework of the SM. In Fig. 5 we display the total (oneloop-corrected) cross section (left panel), together with the relative radiative correction $\delta_{r}$ (right panel), as a function of the SM Higgs-boson mass $M_{\mathrm{H}}$. For completeness, and for illustration purposes, we have also included the scenario corresponding to the last Higgs-boson mass segment ruled out by LEP at a center-of-mass energy $\sqrt{s}=$ $200 \mathrm{GeV}$ (see the leftmost vertical band in that figure). The corresponding production rates for the ILC at $\sqrt{s}=$ $500 \mathrm{GeV}$ and $\sqrt{s}=1 \mathrm{TeV}$ are smaller than in the LEP case due to the suppression of the $s$-channel amplitude by the $Z$-boson propagator at higher energies, see Eq. (5), and the larger mass of the produced Higgs boson. Still, the cross sections for producing SM Higgs bosons of a few hundred $\mathrm{GeV}$ at the startup ILC energy $(\sqrt{s}=500 \mathrm{GeV})$ lie at the $40-60 \mathrm{fb}$ level, which leads roughly to $\sim 25000$ Higgs events for an expected integrated luminosity of $500 \mathrm{fb}^{-1}$. In turn, the one-loop radiative corrections may be either positive or negative, depending on the center-ofmass energy, and lie generally at the level of a few percent, as can be seen in the right panel of Fig. 5. In this panel, we present the evolution of the correction parameter $\delta_{r}$ defined in Eq. (21), as a function of $M_{\mathrm{H}}$. The three peaks (also barely seen in the left panel) are correlated to the production thresholds of $\mathrm{WW}^{-}, \mathrm{Z}^{0} \mathrm{Z}^{0}$, and $\mathrm{tt}$ pairs.

In Figs. 6-9 we illustrate the fundamental phenomenological features associated to the process $\mathrm{e}^{+} \mathrm{e}^{-} \rightarrow \mathrm{h}^{0} \mathrm{Z}^{0}$ in the scope of the general 2HDM. Figure 6 summarizes the pattern of radiative corrections $\delta_{r}$ projected onto the $\left(\tan \beta, \lambda_{5}\right)$ and $(\sin \alpha, \tan \beta)$ planes, the former at fixed $\alpha=$ $\beta-\pi / 2$ and the latter with $\lambda_{5}=-2$. We remark that for $\alpha=\beta-\pi / 2$ the $\mathrm{h}^{0} \mathrm{Z}^{0} \mathrm{Z}^{0}$ tree-level coupling takes on the SM form and therefore is maximal. For definiteness, these plots have been generated for a Higgs-boson mass spectrum as in set B (cf. Table I), and at the fiducial ILC startup center-of-mass energy, $\sqrt{s}=500 \mathrm{GeV}$. Although the range $\tan \beta \gtrsim 1$ is usually the preferred one from the theoretical point of view, we entertain the possibility that $\tan \beta$ can be slightly below 1 in order to better assess the behavior around this value. Figure 6 illustrates that the allowed region in the $\left(\tan \beta, \lambda_{5}\right)$ plane is severely restrained by the theoretical constraints stemming from the perturbative unitarity and vacuum stability. On the one hand, $\lambda_{5}>$ 0 values are strongly disfavored by the vacuum stability condition; on the other hand, the unitarity constraints tend to disfavor moderate and large values of $\tan \beta$ (especially $\tan \beta$ values significantly larger than 1) as well as of 

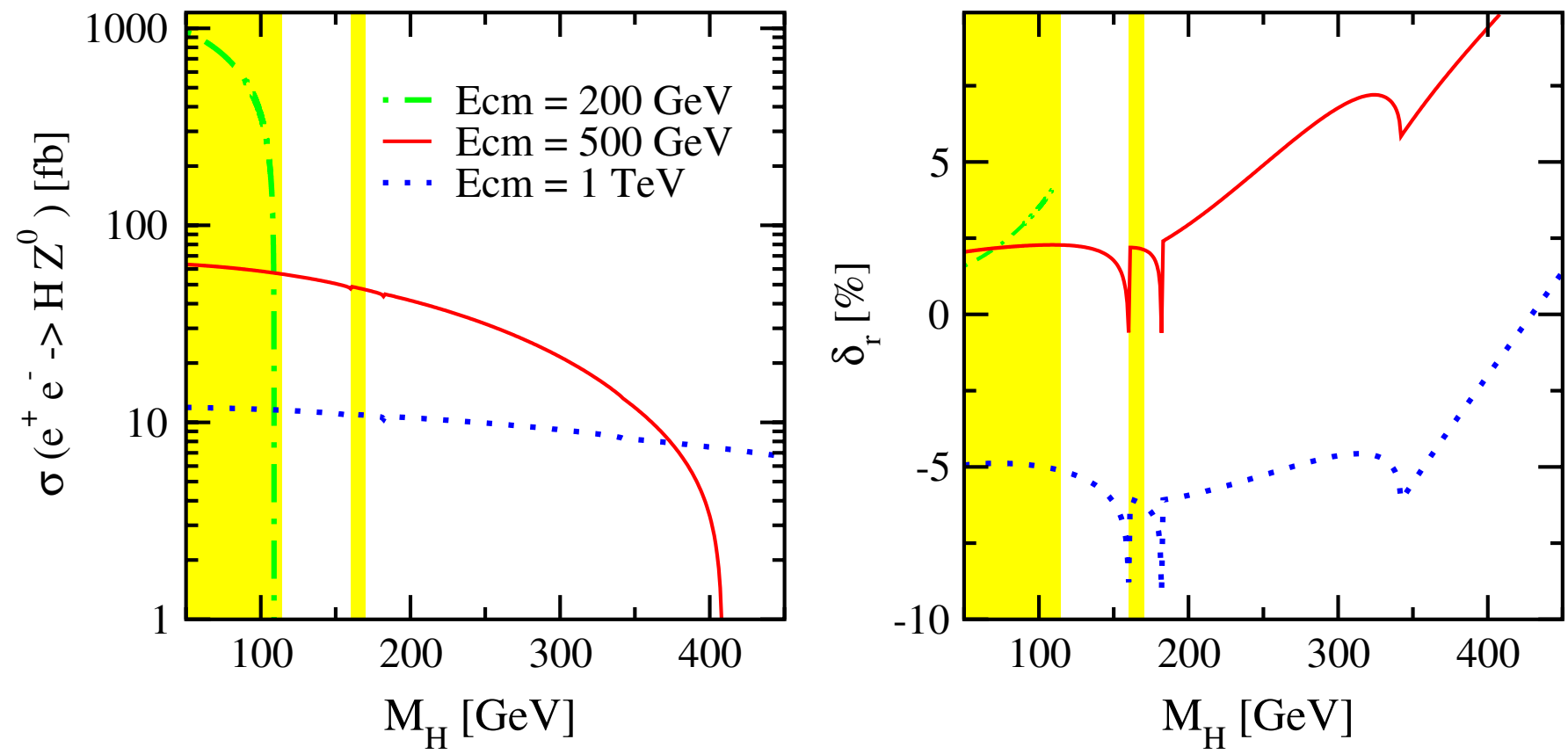

FIG. 5 (color online). Total cross section $\sigma\left(\mathrm{e}^{+} \mathrm{e}^{-} \rightarrow \mathrm{HZ}^{0}\right)$ (in fb) at one-loop (left panel) and relative one-loop correction $\delta_{r}$ (right panel) for $\sqrt{s}=500 \mathrm{GeV}$ and $1 \mathrm{TeV}$, as a function of $M_{\mathrm{H}}$ and within the SM. In the leftmost side band we have computed also the curve corresponding to LEP 200, which falls abruptly from rather high values down until reaching the end of phase space. The thin vertical band corresponds to the narrow exclusion region $160 \lesssim M_{H} \lesssim 170 \mathrm{GeV}$ determined by the Tevatron collaborations [60].

$\tan \beta \ll 1$. Altogether these constraints set an approximate lower bound of $\lambda_{5} \sim-10$ for $\tan \beta \sim 1$ and a rigid upper bound excluding almost all positive values of $\lambda_{5}$ for any $\tan \beta$. The combined set of constraints builds up a characteristic physical domain, with a valley-shaped area centered at $\tan \beta \sim 1$ that sinks into the $\lambda_{5}<0$ region and becomes narrower with growing $\left|\lambda_{5}\right|$.

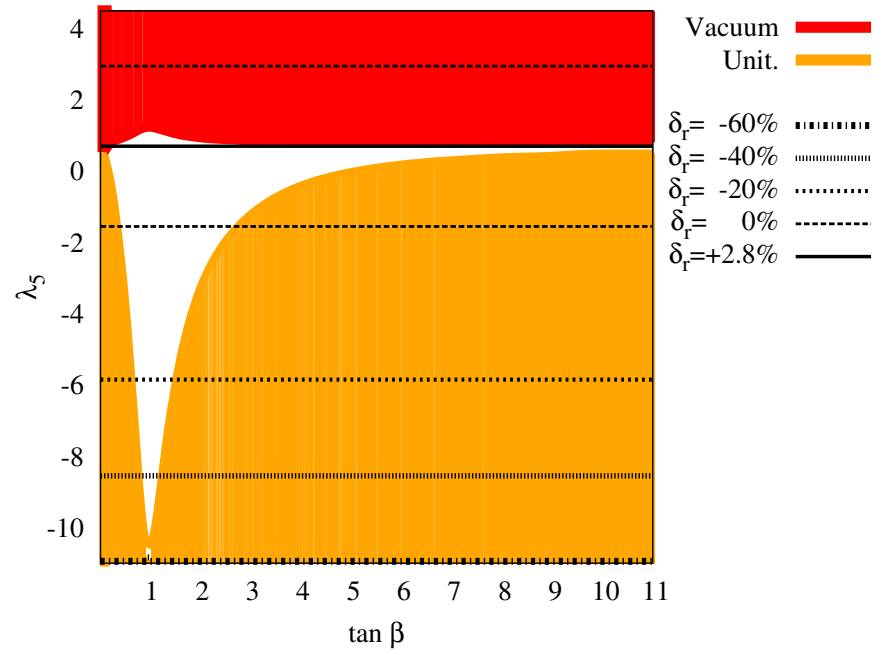

The fact that the curves of constant $\delta_{r}$ do not depend on $\tan \beta$ (left panel of the Fig. 6) is related to the choice $\alpha=$ $\beta-\pi / 2$, which we have made in order to consider the situation where the tree-level cross section for $\mathrm{h}^{0} \mathrm{Z}^{0}$ production is maximal. For this choice of the mixing angle $\alpha$, it turns out that no particular enhancement shows up for any value of $\tan \beta$, neither from the $3 \mathrm{H}$ self-couplings nor from the Higgs-top quark Yukawa couplings. Indeed, under

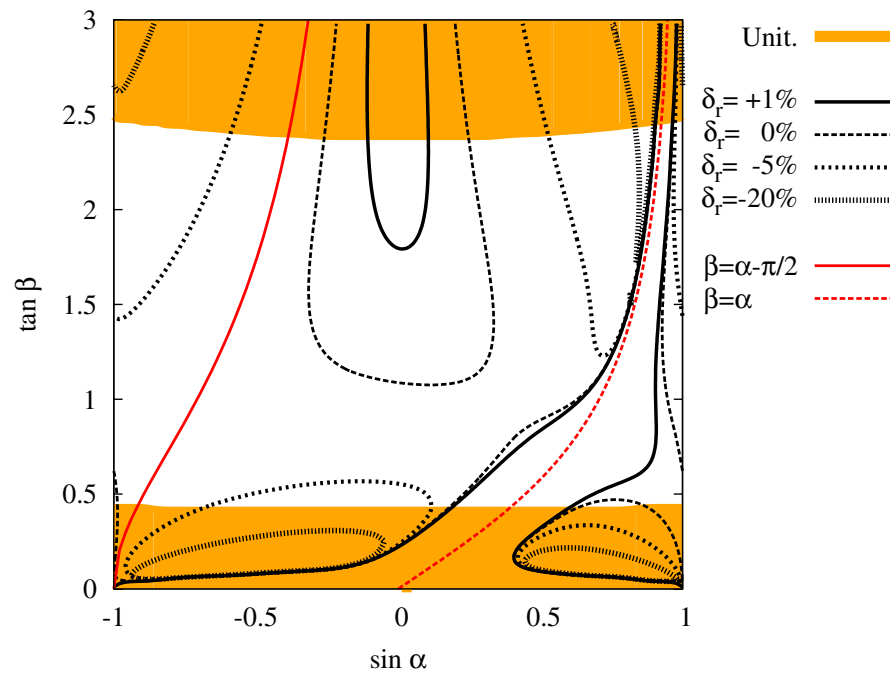

FIG. 6 (color online). Contour lines for the ratio $\delta_{r}$ in the $\left[\tan \beta, \lambda_{5}\right]$ plane assuming $\alpha=\beta-\pi / 2$ (left panel); similarly, in the $[\sin \alpha, \tan \beta]$ plane for $\lambda_{5}=-2$ (right panel). The dark gray shaded areas stand for the regions excluded by the vacuum stability bounds, whereas the light gray shaded areas signal the domains excluded by the perturbative unitarity bounds. The results are obtained for $\sqrt{s}=500 \mathrm{GeV}$ and the Higgs-boson masses as in set B of Table I. 

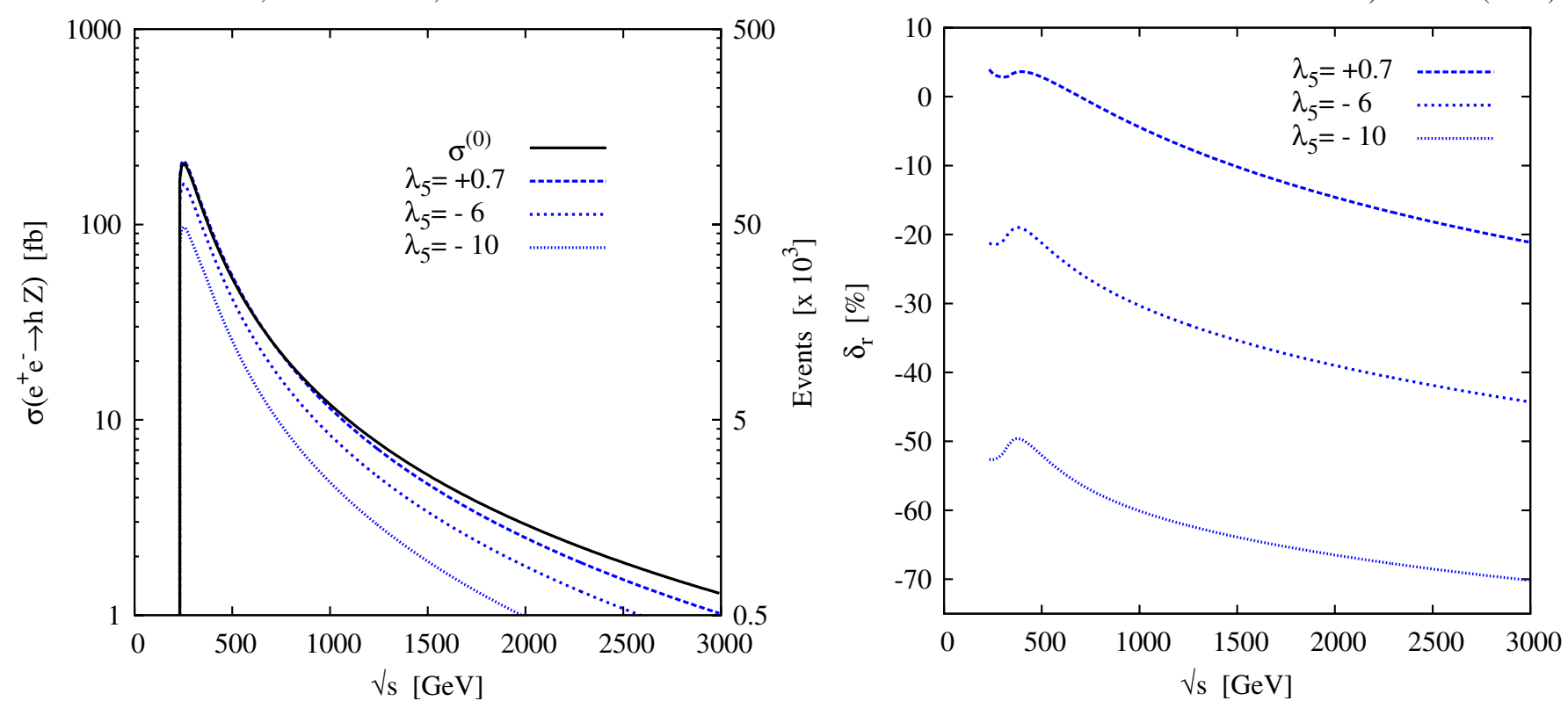

FIG. 7 (color online). Total cross section $\sigma\left(\mathrm{e}^{+} \mathrm{e}^{-} \rightarrow \mathrm{h}^{0} \mathrm{Z}^{0}\right.$ ) (in fb, left panel) and relative one-loop correction $\delta_{r}$ (in $\%$, right panel) as a function of $\sqrt{s}$ using set B of Higgs-boson masses (cf. Table I); for $\tan \beta=1, \alpha=\beta-\pi / 2$ and three different values of $\lambda_{5}$. Shown are also (cf. the right vertical axis of the left panel) the number of events per $500 \mathrm{fb}^{-1}$ of integrated luminosity.

the condition $\alpha=\beta-\pi / 2$, the $\mathrm{h}^{0} \overline{\mathrm{t}}$ Yukawa coupling does not depend on $\tan \beta$ :

$$
\left.\lambda_{\mathrm{h}^{0} \mathrm{Tt}}\right|_{\alpha=\beta-(\pi / 2)}=\left.\frac{e \cos \alpha m_{t}}{2 M_{W} s_{W} \sin \beta}\right|_{\alpha=\beta-(\pi / 2)}=-\frac{e m_{t}}{2 M_{W} s_{W}},
$$

and at the same time the $3 \mathrm{H}$ self-couplings which are relevant for this channel become independent of $\tan \beta$; notice, for example, that

$\left.\lambda_{\mathrm{h}^{0} \mathrm{H}^{0} \mathrm{H}^{0}}\right|_{\alpha=\beta-\pi / 2}=\frac{i e}{2 M_{W} s_{W}}\left[\left(M_{\mathrm{h}^{0}}^{2}+2 M_{\mathrm{H}^{0}}^{2}\right)-\frac{4 \lambda_{5} M_{W}^{2} s_{W}^{2}}{e^{2}}\right]$.

The final outcome is that the potential dependence of the computed observables on $\tan \beta$ vanishes as long as we stick to these $\alpha=\beta-\pi / 2$ configurations-in which the treelevel coupling $\mathrm{h}^{0} \mathrm{Z}^{0} \mathrm{Z}^{0}$ is maximum and formally equivalent to that of the SM. ${ }^{3}$ As a result, the only feasible mechanism able to significantly enhance the quantum effects in these scenarios is by increasing the value of the $\left|\lambda_{5}\right|$ parameter (towards more negative values, so as to be consistent with vacuum stability).

Once we depart from the $\alpha=\beta-\pi / 2$ setting, we recover of course the expected dependence of the computed observables with $\tan \beta$ (cf. right panel of Fig. 6), but then the lowest order $h^{0} Z^{0}$ production cross section becomes smaller. For $\tan \beta<1$, radiative corrections are boosted as a result of the enhanced $3 \mathrm{H}$ self-couplings,

\footnotetext{
${ }^{3}$ An equivalent discussion would hold for the $\mathrm{H}^{0} \mathrm{Z}^{0}$ channel, under the complementary condition $\alpha=\beta$.
}

and partially also due to the Higgs-top quark Yukawa couplings. Either way, their overall effect is to suppress the tree-level signal, as such leading corrections are triggered primordially by the finite Higgs-boson WF corrections. Another source of enhancement of $\delta_{r}$ appears near the region where $\beta \sim \alpha$. However, this effect is not caused by a real increment of the one-loop cross section $\sigma^{(0+1)}$, but by a mere suppression of the cross section at the Born level, i.e. $\sigma^{(0)}$ in the denominator of Eq. (21), and in this sense it is an uninteresting situation.

In Fig. 7 we explore the evolution of the cross section as a function of the center-of-mass energy. We include, in each plot, the tree-level contribution $\sigma^{(0)}$ and the corresponding loop-corrected value, $\sigma^{(0+1)}$, for different values of $\lambda_{5}$. The right panel tracks the related behavior of the quantum correction $\delta_{r}$ as a function of $\sqrt{s}$, for the same set of $\lambda_{5}$ values. The plots are generated for set B of Higgsboson masses, at fixed $\tan \beta=1$ and $\alpha=\beta-\pi / 2$. The leading-order cross section $\sigma^{(0)}$ curve exhibits the expected behavior with $\sqrt{s}$, as it scales with the $s$ channel $Z^{0}$-boson propagator, namely, proportional to $1 /\left(s-M_{Z}^{2}\right)$. A similar pattern is also followed by the full loop-corrected cross section.

The range where the relative one-loop correction $\delta_{r}$ is positive is very reduced and it is confined to a regime where the center-of-mass energy is around the startup value for the ILC $(\sqrt{s} \gtrsim 500 \mathrm{GeV})$ and where $\lambda_{5}$ adopts the (small) positive values allowed by the constraints. It should not come as a surprise that this behavior is similar to the SM result found in Fig. 5; indeed, being $\lambda_{5}$ small there cannot be significant 2HDM enhancements (not even from $\tan \beta$, which is 1) with respect to the corresponding SM 

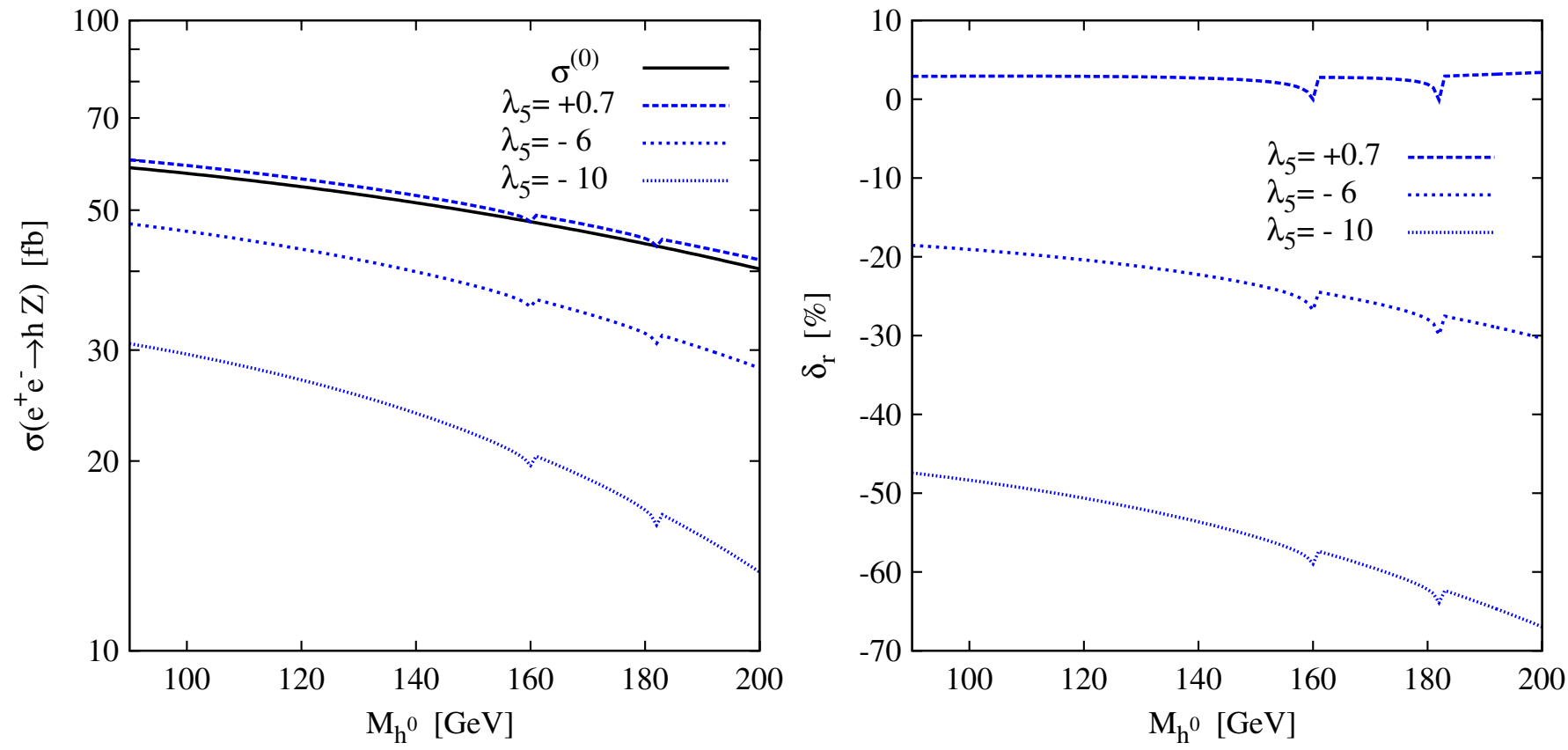

FIG. 8 (color online). Total cross section $\sigma\left(\mathrm{e}^{+} \mathrm{e}^{-} \rightarrow \mathrm{h}^{0} \mathrm{Z}^{0}\right)$ (in fb, left panel) and relative one-loop correction $\delta_{r}$ (right panel) at fixed $\sqrt{s}=500 \mathrm{GeV}$ as a function of $M_{\mathrm{h}^{0}}$ for set B of Higgs-boson masses, cf. Table I. Shown are the results obtained within three different values of $\lambda_{5}$ and for $\alpha=\beta-\pi / 2$.

process. Let us recall that for set $B$ the maximum value of $\lambda_{5}$ allowed by the vacuum stability bounds is $\lambda_{5} \sim 0.65$. Large negative $\lambda_{5}$ values give rise to significant (negative) quantum effects, and translate into loop-corrected cross sections $\sigma^{(0+1)}$ depleted $40 \%$ to $70 \%$ with respect to the tree-level predictions in the entire range from $\sqrt{s}=$ $500 \mathrm{GeV}$ to $\sqrt{s}=3 \mathrm{TeV}$. Since the tree-level $\mathrm{h}^{0} \mathrm{Z}^{0} \mathrm{Z}^{0}$ coupling is equivalent to the SM coupling $\mathrm{HZ}^{0} \mathrm{Z}^{0}$ for $\alpha=$ $\beta-\pi / 2$, the right panel of Fig. 7 also illustrates the departure of the 2HDM loop-corrected cross section with
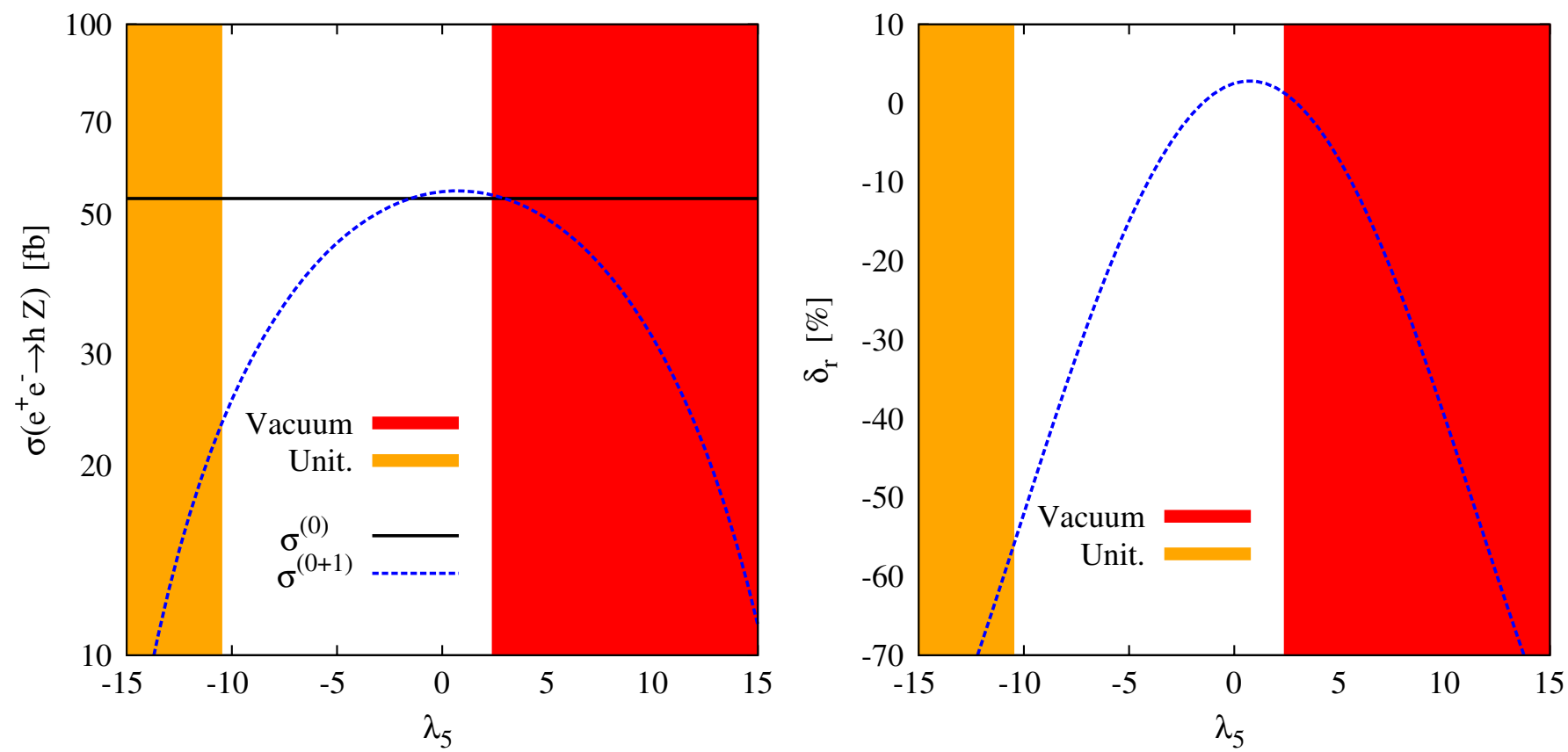

FIG. 9 (color online). Total cross section $\sigma\left(\mathrm{e}^{+} \mathrm{e}^{-} \rightarrow \mathrm{h}^{0} \mathrm{Z}^{0}\right)$ (in fb) at tree level and at one-loop (left panel), together with the relative one-loop correction $\delta_{r}$ (right panel, in $\%$ ) as a function of $\lambda_{5}$. The results are obtained for $\tan \beta=1, \alpha=\beta-\pi / 2, \sqrt{s}=500 \mathrm{GeV}$, and the Higgs-boson masses as in set B of Table I. 
TABLE II. Maximum total cross section $\sigma^{(0+1)}\left(\mathrm{e}^{+} \mathrm{e}^{-} \rightarrow h \mathrm{Z}^{0}\right)$, for $h=\mathrm{h}^{0}, \mathrm{H}^{0}$ at $\sqrt{s}=500 \mathrm{GeV}$, together with the relative size of the radiative corrections $\delta_{r}$, for the different sets of Higgs boson masses quoted in Table I. The results are obtained at fixed tan $\beta=1$ and different values of $\alpha$, with $\lambda_{5}$ at its largest negative attainable value, namely: $\lambda_{5} \simeq-9$ (for set $\mathrm{A}$ ), $\lambda_{5} \simeq-10$ (sets $\mathrm{B}$, C), and $\tan \beta \simeq-8$ (set D).

\begin{tabular}{|c|c|c|c|c|c|c|c|}
\hline & \multicolumn{3}{|c|}{$\mathrm{h}^{0} \mathrm{Z}^{0}$} & \multicolumn{3}{|c|}{$\mathrm{H}^{0} \mathrm{Z}^{0}$} \\
\hline & & $\alpha=\beta-\pi / 2$ & $\alpha=\beta-\pi / 3$ & $\alpha=\beta-\pi / 6$ & $\alpha=\beta-\pi / 3$ & $\alpha=\beta-\pi / 6$ & $\alpha=\beta$ \\
\hline \multirow[t]{2}{*}{ Set A } & $\sigma_{\max }[\mathrm{fb}]$ & 42.61 & 27.89 & 8.17 & 5.36 & 17.86 & 10.70 \\
\hline & $\delta_{r}[\%]$ & -22.79 & -32.62 & -40.76 & -40.92 & -34.40 & -70.51 \\
\hline \multirow[t]{2}{*}{ Set B } & $\sigma_{\max }[\mathrm{fb}]$ & 24.80 & 18.84 & 7.24 & 7.05 & 15.51 & 14.99 \\
\hline & $\delta_{r}[\%]$ & -53.18 & -52.57 & -45.29 & -41.19 & -56.87 & -68.74 \\
\hline \multirow[t]{2}{*}{ Set $C$} & $\sigma_{\max }[\mathrm{fb}]$ & 28.81 & 21.97 & 7.75 & 7.83 & 20.82 & 25.60 \\
\hline & $\delta_{r}[\%]$ & -43.91 & -42.97 & -39.66 & -36.96 & -44.13 & -48.48 \\
\hline \multirow[t]{2}{*}{ Set D } & $\sigma_{\max }[\mathrm{fb}]$ & 40.77 & 29.25 & 9.30 & 8.56 & 23.95 & 28.59 \\
\hline & $\delta_{r}[\%]$ & -17.95 & -21.52 & -25.15 & -15.14 & -20.84 & -29.12 \\
\hline
\end{tabular}

respect to the tree-level SM cross section, namely $\left(\sigma_{2 \mathrm{HDM}}^{(0+1)}-\sigma_{\mathrm{SM}}^{(0)}\right) / \sigma_{\mathrm{SM}}^{(0)}$.

The behavior of $\sigma\left(\mathrm{e}^{+} \mathrm{e}^{-} \rightarrow \mathrm{h}^{0} \mathrm{Z}^{0}\right)$ as a function of the Higgs-boson mass $M_{\mathrm{h}^{0}}$ is presented in Fig. 8. This figure is the 2HDM counterpart of Fig. 5 corresponding to the SM case. We superimpose the tree-level and the loop-corrected cross sections for the set B of Higgs-boson masses, by setting $\tan \beta=1, \alpha=\beta-\pi / 2$ and using three different values of $\lambda_{5}$. The center-of-mass energy is settled at the fiducial value $\sqrt{s}=500 \mathrm{GeV}$. Obviously, the raise of the Higgs-boson mass $M_{\mathrm{h}^{0}}$ implies a reduction of the available phase space, so that the cross section falls down. In the left panel of Fig. 8, both the tree-level and the loop-corrected cross sections decrease monotonically with the growing of $M_{\mathrm{h}^{0}}$. The $\mathrm{Z}^{0} \mathrm{Z}^{0}$ and $\mathrm{W}^{+} \mathrm{W}^{-}$thresholds are also barely visible therein. In the right panel of the same figure we observe a steady increase of the negative value of the correction for heavier Higgs-boson mass, whereas the case with positive correction remains almost stable.

Finally, in Fig. 9 we display the tree-level contribution and the total (one-loop-corrected) cross section (left panel), together with the relative radiative correction $\delta_{r}$ (right panel), as a function of the parameter $\lambda_{5}$. These plots have been generated in the same benchmark conditions as in Fig. 8 and using a fiducial ILC start-up center-of-mass energy $\sqrt{s}=500 \mathrm{GeV}$. Let us remember that the red shaded area on the right-hand side of that figure (specifically for $\lambda_{5} \gtrsim 2$.4) stands for the region excluded by the vacuum stability bounds, whereas the light gray shaded area on the left $\left(\lambda_{5} \lesssim-10.5\right)$ signals the domain excluded by the perturbative unitarity bounds. The cross section is seen to follow the expected behavior, which we have derived from the estimate of the leading effect-cf. Eq. (16) - in combination with the structure of the trilinear coupling (23), namely, a negative quadratic dependence of the scattering amplitude, which translates into $\sigma\left(\mathrm{e}^{+} \mathrm{e}^{-} \rightarrow\right.$ $\left.\mathrm{h}^{0} \mathrm{Z}^{0}\right) \sim\left(a-b \lambda_{5}^{2}\right)^{2}$, emerging ultimately from the finite WF corrections to the external Higgs-boson leg (notice the inclusion of the leading quartic corrections). For moderate negative $\lambda_{5}$, the relative size of the one-loop quantum corrections can reach up to $\sim-50 \%$. Positive $\delta_{r}$, however, can only take place for $\lambda_{5}$ close to zero.

A rather comprehensive survey of the predicted cross sections for different values of the tree-level coupling and different Higgs mass setups is presented in Table II. We display the results for the one-loop-corrected cross sections (in $\mathrm{fb}$ ), together with the relative radiative corrections [cf. Eq. (21)], for both neutral Higgs-strahlung channels $\mathrm{h}^{0} \mathrm{Z}^{0}$ and $\mathrm{H}^{0} \mathrm{Z}^{0}$. Once more, we set $\sqrt{s}=500 \mathrm{GeV}$; and we work at $\tan \beta=1$ and maximum allowed $\left|\lambda_{5}\right|$, since we are mostly interested in spotlighting the imprints of the $3 \mathrm{H}$ self-couplings in the quantum effects associated to the Higgs-strahlung mechanism. Let us also recall in passing that $\alpha=\beta-\pi / 2$ maximizes the tree-level $h^{0} \mathrm{Z}^{0} \mathrm{Z}^{0}$ coupling, while $\mathrm{H}^{0} \mathrm{Z}^{0} \mathrm{Z}^{0}$ is optimal in the complementary regime, $\alpha=\beta$. From the Table we may read out that radiative corrections in these regimes are certainly large, regardless of the details of the chosen mass spectrum, the tree-level coupling, and the actual channel under consideration. In a nutshell, the characteristic signature of the enhanced $3 \mathrm{H}$ self-couplings in the pattern of quantum effects on the Higgs-strahlung processes is rather universal and manifests in the form of a substantial depletion of the tree-level signal (typically in the range of $\delta_{r} \sim-20 /-$ $60 \%$ ). Fortunately, even under such a dramatic suppression the final loop-corrected cross sections stay at the level of a few tens of $\mathrm{fb}$ - thereby amounting to a non-negligible yield of $\sim 10^{3}-10^{4}$ events per $500 \mathrm{fb}^{-1}$.

A note of caution should be given at this point in regard to some particular configurations of the $\mathrm{H}^{0} \mathrm{Z}^{0}$ channel. It turns out that certain choices of Higgs-boson masses (e.g. for MSSM-like mass splittings) may lie close, or simply beyond, the kinematical threshold for the decay process $\mathrm{H}^{0} \rightarrow \mathrm{h}^{0} \mathrm{~h}^{0}$; that is to say, $M \simeq 2 M$. If we then consider a regime wherein $\alpha=\beta$ and sizable $\left|\lambda_{5}\right|$, then the $\mathrm{H}^{0} \mathrm{WF}$ correction undergoes a remarkable boost, which results from the combination of two independent sources of enhancement, namely, (i) the actual strength of the $\mathrm{H}^{0} \mathrm{~h}^{0} \mathrm{~h}^{0}$ 
coupling - which is maximally enlarged in this regimeand (ii) the kinematical enhancement due to the vicinity of the $\mathrm{H}^{0} \rightarrow \mathrm{h}^{0} \mathrm{~h}^{0}$ threshold, which is reflected as a sharp peak in $\delta Z_{\mathrm{H}^{0}} \sim B_{0}^{\prime}\left(M_{\mathrm{H}^{0}}^{2}, M_{\mathrm{h}^{0}}^{2}, M_{\mathrm{h}^{0}}^{2}\right)$. However, these $\mathrm{H}^{0} \mathrm{WF}$ corrections become so large that the perturbative formula (16) is no longer valid and we have to keep the original (unexpanded) expression $\hat{Z}_{\mathrm{H}^{0}}^{1 / 2}=\left[1+\operatorname{Re} \hat{\Sigma}_{\mathrm{H}^{0}}^{\prime}\left(M_{\mathrm{H}^{0}}^{2}\right)\right]^{-1 / 2}$. Out of such a corner in the parameter space, the higher-order effects involved in the above resummed form of the WF corrections become harmless and totally inconspicuous and can be safely discarded. If, alternatively, we were considering a mass setup such that $M_{\mathrm{H}^{0}}>2 M_{\mathrm{h}^{0}}$, then the decay $\mathrm{H}^{0} \rightarrow \mathrm{h}^{0} \mathrm{~h}^{0}$ would be open and, in the scenario of maximum $\mathrm{H}^{0} \mathrm{~h}^{0} \mathrm{~h}^{0}$ coupling, it would furnish a very large width for $\mathrm{H}^{0}$. In this particular setup, the process would effectively boil down to the double Higgs-strahlung channels $\mathrm{e}^{+} \mathrm{e}^{-} \rightarrow \mathrm{H}^{0} \mathrm{Z}^{0} \rightarrow \mathrm{h}^{0} \mathrm{~h}^{0} \mathrm{Z}^{0}$ previously explored in the literature [22].

The following comment is in order to clarify the role played by the remaining (potential) sources of enhancement. We have seen that the various constraints restrict very significantly the range of allowed values of $\tan \beta$, and enforce it to stay around 1 when $\left|\lambda_{5}\right|$ is maximum in the region $\lambda_{5}<0$. As a result, the contributions from the top quark and bottom quark Yukawa couplings cannot be augmented in the domain where the trilinear couplings are maximal. Remarkably enough, let us also emphasize that they cannot be enhanced even in the region where $\left|\lambda_{5}\right|$ is small or zero. To see why, notice that although in such a region the parameter $\tan \beta$ can be very large or small and still be compatible with the constraints (cf. Fig. 6), then all the terms in the trilinear couplings (cf. Table II of Ref. [32]) which are not proportional to $\lambda_{5}$ become of order one (i.e. cannot be promoted to high values) in the regime where the tree-level production cross section for $\mathrm{h}^{0}$ or $\mathrm{H}^{0}$ is optimal. Not only so, in the very same regime the Yukawa couplings of both the top and bottom quarks cannot be enhanced either. One can easily check all these features explicitly by observing that, in the regions where the corresponding tree-level processes are maximal, the $\tan \beta$ enhancements are canceled in all the couplings. We have checked numerically that the residual corrections (positive and negative) are merely of a few percent for any value of $\tan \beta$. At the end of the day, we conclude that the only sizable and eventually measurable quantum effects on the processes under study are those stemming potentially from the enhancement of the $\lambda_{5}$ coupling, not from the Yukawa couplings.

Let us finally emphasize that the complementarity between the neutral final states $\mathrm{h}^{0} \mathrm{Z}^{0} / \mathrm{h}^{0} \mathrm{~A}^{0}$ and $\mathrm{H}^{0} \mathrm{Z}^{0} / \mathrm{H}^{0} \mathrm{~A}^{0}$, i.e. processes (1) and (2), is a unique chance for analyzing potentially big correlations between quantum effects in the $2 \mathrm{HDM}$ production cross sections, as there is no similar opportunity in the charged sector. Indeed, the final state charged counterparts $\mathrm{H}^{ \pm} \mathrm{W}^{\mp}$ are suppressed owing to the fact that the $\mathrm{ZH}^{ \pm} \mathrm{W}^{\mp}$ vertices are forbidden at the tree level in any 2HDM extension of the SM [55] and therefore they can only be studied in more general extensions of the Higgs sector [56], or through loop-induced $\mathrm{H}^{ \pm} \mathrm{W}^{\mp}$ vertices in the 2HDM [57] and in the MSSM [58]. In these loop-induced mechanisms, the cross section for the associated $\mathrm{H}^{ \pm} \mathrm{W}^{\mp}$ production in a linear collider is rather meager-generally below $1 \mathrm{fb}$ at the startup ILC energy (for charged Higgs masses comparable to the ones we have considered for the $\mathrm{h}^{0} \mathrm{Z}^{0} / \mathrm{h}^{0} \mathrm{~A}^{0}$ production) and within the region allowed by the current constraints. We therefore deem quite difficult to use the $\mathrm{H}^{ \pm} \mathrm{W}^{\mp}$ channel to extract additional information. In our opinion, the main task should be focused on performing precision tests of the $\mathrm{h}^{0} \mathrm{Z}^{0} / \mathrm{h}^{0} \mathrm{~A}^{0}$ final states, together with the $\mathrm{H}^{0} \mathrm{Z}^{0} / \mathrm{H}^{0} \mathrm{~A}^{0}$ ones (if the mass of the heavy $\mathcal{C P}$-even Higgs is not too large).

\section{DISCUSSION AND CONCLUSIONS}

In this article, we have concentrated on the analysis of the production of neutral Higgs bosons in association with the $Z^{0}$ gauge boson at the future linac facilities within the framework of the general (nonsupersymmetric) twoHiggs-doublet model (2HDM). Our basic endeavor has been twofold: (i) on the one hand to study the impact of radiative corrections to the final predicted rates; and (ii) on the other hand to correlate such quantum effects with the enhancement potential of the $3 \mathrm{H}$ self-interactions, which are a genuine dynamical feature of the $2 \mathrm{HDM}-$ in the sense that it is unmatched to its supersymmetric counterpart. The upshot of our analysis singles out sizable (although negative) quantum effects which are correlated to the enhancement properties of the $3 \mathrm{H}$ self-interactions in the general 2HDM. Such large effects are identified fundamentally in the region of parameter space with $\tan \beta \simeq 1$ and where the coupling $\left|\lambda_{5}\right|$ is at its maximum possible value compatible with the various constraints. The quantum effects reach typically $\delta \sigma / \sigma \sim-20 \% /-60 \%$, for $\left|\lambda_{5}\right| \sim 8 / \sim 10\left(\lambda_{5}<0\right)$, this being the crucial parameter that tunes the actual size of the $3 \mathrm{H}$ self-couplings. Let us stress that the most stringent limits on $\lambda_{5}$ are placed by the conditions of perturbative unitarity and vacuum stability. ${ }^{4}$

Although the vertex corrections to the $\mathrm{h}^{0} \mathrm{Z}^{0} \mathrm{Z}^{0} / \mathrm{H}^{0} \mathrm{Z}^{0} \mathrm{Z}^{0}$ couplings are sensitive to such $3 \mathrm{H}$ self-interactions through Higgs-mediated one-loop diagrams, the most significant

\footnotetext{
${ }^{4}$ Recently, a combined analysis of different $B$-meson physics constraints over the 2 HDM parameter space suggests that values of $\tan \beta \sim 1$ could be disfavored for charged Higgs masses $M_{\mathrm{H}^{ \pm}}$ too near to the lowest mass limit of $300 \mathrm{GeV}$ (and certainly below) [59]. However, the level of significance is not high and, in addition, our leading quantum corrections are basically insensitive to the charged Higgs mass. Therefore, a shift of $M_{\mathrm{H}^{ \pm}}$slightly upwards restores the possibility of $\tan \beta \sim 1$ at, say, $2 \sigma$ without altering significantly our results (as we have explicitly checked).
} 
quantum effects are induced by the wave-function renormalization corrections to the $\mathrm{h}^{0} / \mathrm{H}^{0}$ Higgs-boson external lines. These (negative) effects triggered by the trilinear couplings are of order $\alpha_{\mathrm{ew}} \lambda_{3 H}^{2}$, and are therefore very responsive to changes in the value of the parameter $\lambda_{5}$ in the Higgs potential of the 2HDM. In turn, the gauge-boson and fermion one-loop contributions remain subleading (including the effects from the Yukawa couplings) as they show no remarkable departures from their SM analogues in the relevant regions of parameter space-which yield $\delta \sigma / \sigma$ at the level of a few percent. Most important is also the fact that the combined analysis of such Higgsstrahlung events with the previously considered Higgs-pair production processes $\left(\mathrm{h}^{0} \mathrm{~A}^{0}, \mathrm{H}^{0} \mathrm{~A}^{0}\right)$ - see [32] for detailscould be instrumental as a highly sensitive probe of the underlying architecture of the Higgs sector. At the fiducial center-of-mass energy value of $\sqrt{s}=500 \mathrm{GeV}$, and when the genuine enhancement mechanisms of the 2HDM are active, the $h \mathrm{~A}^{0}$ events are remarkably strengthened at the one-loop level while the $h \mathrm{Z}^{0}$ channels are simultaneously depleted.

We can assert that the described pattern of leading quantum effects emerges as a kind of "universal" feature of the 2HDM as far as the predictions for the Higgsstrahlung processes are concerned, meaning that this pattern is virtually independent of the details of the Higgs mass spectrum and of the actual production channel $\left(\mathrm{h}^{0} \mathrm{Z}^{0}, \mathrm{H}^{0} \mathrm{Z}^{0}\right)$. The same is true for the pairwise production channels [32]. Therefore they all can be physically significant provided the produced Higgs boson is not too heavy for the actual center-of-mass energy. Focusing on the Higgs-strahlung processes, the rise of large (and negative) radiative corrections to their cross sections within the $2 \mathrm{HDM}$ can be regarded as a characteristic imprint of a general two-Higgs-doublet model structure. Furthermore, the presence of a $Z^{0}$ boson in the final state (and hence of its clear-cut leptonic signature $Z^{0} \rightarrow l^{+} l^{-}$) should enable a rather comfortable tagging of these Higgs events following a method entirely similar to the original Bjorken mechanism [35] in the SM. Although the corresponding cross sections at the higher operating energies planned for the future linear colliders are smaller (as compared to LEP), they are nevertheless sufficiently sizable (typically in the range $10-40 \mathrm{fb}$ ) for a relatively comfortable practical measurement at the higher luminosities scheduled for these machines. For example, the associated decay of the accompanying neutral $\mathcal{C P}$-even Higgs boson would manifest basically in the form of either (i) collimated and highly energetic $b$-quark or $\tau$-lepton jets, for $M_{\mathrm{h}^{0}} \lesssim 2 M_{V} \sim 180 \mathrm{GeV}$; and (ii) $\mathrm{h}^{0}, \mathrm{H}^{0} \rightarrow$ $\mathrm{W}^{+} \mathrm{W}^{-} \rightarrow l^{+} l^{-}+$missing energy; $\quad$ or $\quad \mathrm{H}^{0} \rightarrow \mathrm{Z}^{0} \mathrm{Z}^{0} \rightarrow$ $l^{+} l^{-} l^{\prime+} l^{\prime-}$, for $M_{\mathrm{H}^{0}}>2 M_{V}$ (in the optimal regime for the tree-level process).

Some discussion on how the corresponding MSSM Higgs-boson production cross sections compare to the
2HDM ones seems also appropriate. In the MSSM, the maximum quantum effects on $h Z^{0}$ production are typically milder. Here, the dominance of the WF corrections is also the main source of one-loop effects [29]. They are usually reabsorbed into the tree-level ZZh couplings (4), specifically in the $\mathcal{C} \mathcal{P}$-even mixing angle $\alpha$, which then becomes an effective mixing parameter. Consider, for example, the behavior of the characteristic trigonometric coupling $\cos ^{2}(\beta-\alpha)$ in the $\mathrm{ZZH}^{0}$ interaction vertex, which in the MSSM dies away with growing values of $M_{\mathrm{A}^{0}}$ (both at the tree level and at one-loop). It follows that in a situation where $M_{\mathrm{A}^{0}}$ is sufficiently heavy, say above $200 \mathrm{GeV}$, the MSSM would predict measurable rates for the channel $\mathrm{h}^{0} \mathrm{Z}^{0}$ (and also for $\mathrm{H}^{0} \mathrm{~A}^{0}$, if $\sqrt{s}$ is sufficiently high), whereas the complementary channels $\left(\mathrm{H}^{0} \mathrm{Z}^{0} / \mathrm{h}^{0} \mathrm{~A}^{0}\right)$ would be virtually below the observability threshold. In contrast, in this particular SM-like regime [in which $\sin (\beta-\alpha) \rightarrow$ 1] the $h^{0} Z^{0}$ cross section in the $2 \mathrm{HDM}$ could well be exhibiting the trademark suppression induced at one-loop by large $3 \mathrm{H}$ self-couplings.

Furthermore, in the MSSM, the dynamical origin of the leading WF effects does not reside in the Higgs-boson selfcouplings, but on the Yukawa couplings with fermions and also in the large Yukawa-like couplings of Higgs bosons with sfermions, particularly with the stop. Thus, in both models (2HDM or MSSM) the main source of quantum effects emanates from the renormalization of the Higgsboson external lines, but the kind of interactions involved in each case is radically different. On the quantitative side, the impact of the quantum effects in the MSSM case turns out to be considerably milder and as a consequence the cross sections for Higgs-strahlung production remain similar to the SM cross section (i.e. for the Bjorken process). The corresponding $2 \mathrm{HDM}$ cross sections, instead, can be significantly smaller-if taking the same Higgs-boson masses (e.g. sets A and B of Table I, which mimic the MSSM Higgs-boson mass spectrum) owing to the aforementioned large suppression effect from the Higgs-boson WF renormalization. At the same time, as we have already mentioned, the cross sections for neutral Higgs-boson pair production in the general $2 \mathrm{HDM}$-cf. Eq. (1)—become substantially larger than the MSSM counterparts (for the same Higgs-boson mass spectrum), carrying very sizable and positive quantum effects [32]. Therefore, an interesting combined picture emerges in the 2HDM context, in which a large $(\sim 50 \%)$ enhancement of the pairwise neutral Higgs-boson production is simultaneously accompanied by a drastic drop (of similar size) of the Higgs-strahlung events. As this situation is completely impossible to realize in the MSSM, this feature could be used as a strong characteristic signature to discriminate between these processes in the general 2HDM and in the MSSM. In other words, it could provide an essential quantum handle enabling us to perform a proper identification of the kind of Higgs bosons produced in a linear collider. 
In summary, we have analyzed the classical Higgs-boson-strahlung processes at linear colliders in the light of the general 2HDM, and we have elucidated very significant quantum imprints which spotlight, once more, the stupendous phenomenological possibilities that triple Higgsboson self-interactions could encapsulate in nonsupersymmetric extended Higgs sectors. To be sure, regardless of whether the LHC is finally capable to discover the Higgs boson(s), a paramount effort will still be mandatory in order to completely settle its experimental basis and to uncover the nature of the spinless constituents behind the electroweak symmetry-breaking mechanism. As we have shown, experiments at the future linear collider facilities could play a crucial role in this momentous endeavor.

\section{ACKNOWLEDGMENTS}

N.B. thanks an ER position of the EU Project No. MRTN-CT-2006-035505 HEPTools and the hospitality at the Departament ECM of the Universitat de Barcelona; D. L. V. acknowledges the MEC FPU Grant No. AP2006-00357. The work of J. S. has been supported in part by MEC and FEDER under Project No. FPA200766665 and by DIUE/CUR Generalitat de Catalunya under Project No. 2009SGR502 and by the Spanish ConsoliderIngenio 2010 program CPAN CSD2007-00042. D. L. V. wishes to thank the hospitality of the Theory Group at the Physikalisches Institut of the University of Bonn and the Bethe Center for Theoretical Physics. Discussions with Karina E. Williams are gratefully acknowledged.
[1] P. W. Higgs, Phys. Lett. 12, 132 (1964); Phys. Rev. Lett. 13, 508 (1964); F. Englert and R. Brout, Phys. Rev. Lett. 13, 321 (1964); G. S. Guralnik, C. R. Hagen, and T. W. B. Kibble, Phys. Rev. Lett. 13, 585 (1964).

[2] H. P. Nilles, Phys. Rep. 110, 1 (1984); H. E. Haber and G. L. Kane, Phys. Rep. 117, 75 (1985); Supersymmetry, edited by S. Ferrara (North-Holland/World Scientific, Singapore, 1987), Vol. 1-2.

[3] J. A. Coarasa, D. García, J. Guasch, R. A. Jiménez, and J. Solà, Eur. Phys. J. C 2, 373 (1998); Phys. Lett. B 425, 329 (1998).

[4] R. A. Jiménez and J. Solà, Phys. Lett. B 389, 53 (1996); J. A. Coarasa, R. A. Jiménez, and J. Solà, Phys. Lett. B 389, 312 (1996); J. Guasch, R. A. Jiménez, and J. Solà, Phys. Lett. B 360, 47 (1995); D. García, R. A. Jiménez, J. Solà, and W. Hollik, Nucl. Phys. B427, 53 (1994).

[5] J. Guasch, W. Hollik, and J. Solà, J. High Energy Phys. 10 (2002) 040; Phys. Lett. B 510, 211 (2001); Phys. Lett. B 437, 88 (1998).

[6] M. S. Carena, D. García, U. Nierste, and C. E. M. Wagner, Nucl. Phys. B577, 88 (2000); Phys. Lett. B 499, 141 (2001).

[7] A. Belyaev, D. García, J. Guasch, and J. Solà, Phys. Rev. D 65, 031701 (2002); J. High Energy Phys. 06 (2002) 059.

[8] M. S. Carena and H. E. Haber, Prog. Part. Nucl. Phys. 50, 63 (2003).

[9] A. Djouadi, Phys. Rep. 459, 1 (2008); Phys. Rep. 457, 1 (2008).

[10] S. Heinemeyer, Acta Phys. Pol. B 39, 2673 (2008); arXiv:0912.0361.

[11] J. F. Gunion, H. E. Haber, G. L. Kane, and S. Dawson, The Higgs Hunter's Guide (Addison-Wesley, Menlo Park, CA, 1990).

[12] J. A. Coarasa, J. Guasch, J. Solà, and W. Hollik, Phys. Lett. B 442, 326 (1998).

[13] S. Béjar, J. Guasch, and J. Solà, Nucl. Phys. B600, 21 (2001); Nucl. Phys. B675, 270 (2003).

[14] J. Guasch and J. Solà, Nucl. Phys. B562, 3 (1999).

[15] S. Béjar, J. Guasch, D. López-Val, and J. Solà, Phys. Lett.
B 668, 364 (2008); D. López-Val, J. Guasch, and J. Solà, J. High Energy Phys. 12 (2007) 054; J. Guasch, W. Hollik, S. Peñaranda, and J. Solà, Nucl. Phys. Proc. Suppl. 157, 152 (2006); G. Eilam, M. Frank, and I. Turan, Phys. Rev. D 74, 035012 (2006).

[16] P. Draper, T. Liu, and C. E. M. Wagner, Phys. Rev. D 80, 035025 (2009).

[17] A. Arhrib, R. Benbrik, C.-H. Chen, R. Guedes, and R. Santos, J. High Energy Phys. 08 (2009) 035.

[18] G. Weiglein et al., Phys. Rep. 426, 47 (2006).

[19] For the ILC, see http://www.linearcollider.org/cms/; for CLIC, see http://clic-study.web.cern.ch/clic-study/.

[20] A. Djouadi, W. Kilian, M. Mühlleitner, and P. M. Zerwas, Eur. Phys. J. C 10, 45 (1999); M. Mühlleitner, arXiv:hepph/0008127.

[21] G. Ferrera, J. Guasch, D. López-Val, and J. Solà, Phys. Lett. B 659, 297 (2008); Proc Sci., RADCOR2007 (2007) 043.

[22] A. Arhrib, R. Benbrik, and C.-W. Chiang, Phys. Rev. D 77, 115013 (2008); AIP Conf. Proc. 1006, 112 (2008).

[23] R. N. Hodgkinson, D. López-Val, and J. Solà, Phys. Lett. B 673, 47 (2009).

[24] M. Krawczyk, arXiv:hep-ph/0307314.

[25] N. Bernal, D. López-Val, and J. Solà, Phys. Lett. B 677, 39 (2009); P. Posch, arXiv:1001.1759.

[26] F. Cornet and W. Hollik, Phys. Lett. B 669, 58 (2008); E. Asakawa, D. Harada, S. Kanemura, Y. Okada, and K. Tsumura, Phys. Lett. B 672, 354 (2009); A. Arhrib, R. Benbrik, C.-H. Chen, and R. Santos, Phys. Rev. D 80, 015010 (2009).

[27] P. Chankowski, S. Pokorski, and J. Rosiek, Nucl. Phys. B423, 437 (1994); P. H. Chankowski and S. Pokorski, Phys. Lett. B 356, 307 (1995).

[28] V. Driesen and W. Hollik, Z. Phys. C 68, 485 (1995); V. Driesen, W. Hollik, and J. Rosiek, Z. Phys. C 71, 259 (1996); arXiv:hep-ph/9605437.

[29] S. Heinemeyer, W. Hollik, J. Rosiek, and G. Weiglein, Eur. Phys. J. C 19, 535 (2001).

[30] J. L. Feng and T. Moroi, Phys. Rev. D 56, 5962 (1997); E. 
Coniavitis and A. Ferrari, Phys. Rev. D 75, 015004 (2007).

[31] A. Arhrib and G. Moultaka, Nucl. Phys. B558, 3 (1999); A. Kraft, Ph.D. thesis, Universität Karlsruhe, 1999; J. Guasch, W. Hollik, and A. Kraft, Nucl. Phys. B562, 3 (1999); arXiv:hep-ph/9911452.

[32] D. López-Val and J. Solà, Phys. Rev. D 81, 033003 (2010). For a summarized presentation, see D. López-Val and J. Solà, Proc. Sci., RADCOR2009 (2010) 045.

[33] J. F. Gunion, H. E. Haber, and J. Wudka, Phys. Rev. D 43, 904 (1991).

[34] A. Djouadi, H. E. Haber, and P. M. Zerwas, Phys. Lett. B 375, 203 (1996); A. Djouadi, V. Driesen, W. Hollik, and J. Rosiek, Nucl. Phys. B491, 68 (1997);

[35] J. D. Bjorken, in Proceedings of the 1976 SLAC Summer Institute on Particle Physics, edited by M. Zipf (SLAC Report No. 198, 1976), p. 22; D. R. T. Jones and S. T. Petcov, Phys. Lett. 84B, 440 (1979).

[36] C. Amsler et al. (The Particle Data Group), Phys. Lett. B 667, 1 (2008).

[37] E. Gross, G. Wolf, and B. A. Kniehl, Z. Phys. C 63, 417 (1994); 66, 321(E) (1995); G. Altarelli, T. Sjöstrand, and F. Zwirner, CERN Report No. 96-01 (1996).

[38] A. Djouadi, J. Kalinowski, and P. M. Zerwas, Z. Phys. C 57, 569 (1993); P. H. Chankowski, S. Pokorski, and J. Rosiek, Nucl. Phys. B423, 497 (1994); V. Driesen and W. Hollik, Z. Phys. C 68, 485 (1995); A. Djouadi, J. Kalinowski, P. Ohmann, and P. Zerwas, Z. Phys. C 74, 93 (1997).

[39] K. E. Williams, G. Weiglein (to be published).

[40] M. Frank, T. Hahn, S. Heinemeyer, W. Hollik, H. Rzehak, and G. Weiglein, J. High Energy Phys. 02 (2007) 047.

[41] M. B. Einhorn, D. R. T. Jones, and M. J. G. Veltman, Nucl. Phys. B123, 89 (1977).

[42] M. Misiak and M. Steinhauser, Nucl. Phys. B764, 62 (2007); M. Misiak et al., Phys. Rev. Lett. 98, 022002 (2007).

[43] V. D. Barger, J. L. Hewett, and R. J. N. Phillips, Phys. Rev. D 41, 3421 (1990); A. G. Akeroyd, Phys. Lett. B 368, 89 (1996).

[44] S. Kanemura, T. Kubota, and E. Takasugi, Phys. Lett. B 313, 155 (1993); A. Arhrib, arXiv:hep-ph/0012353; A. G. Akeroyd, A. Arhrib, and E.-M. Naimi, Phys. Lett. B 490,
119 (2000).

[45] M. Sher, Phys. Rep. 179, 273 (1989); S. Nie and M. Sher, Phys. Lett. B 449, 89 (1999).

[46] D. Kominis and R. S. Chivukula, Phys. Lett. B 304, 152 (1993); S. Kanemura, T. Kasai, and Y. Okada, Phys. Lett. B 471, 182 (1999).

[47] K. Aoki, Z. Hioki, M. Konuma, R. Kawabe, and T. Muta, Prog. Theor. Phys. Suppl. 73, 1 (1982); M. Bohm, H. Spiesberger, and W. Hollik, Fortsch. Phys. 34, 687 (1986); A. Sirlin, Phys. Rev. D 22, 971 (1980); W. Hollik, Precision Tests of the Standard Electroweak Model, edited by P. Langacker, Advanced Series on Directions in High Energy Physics Vol. 14 (World Scientific, Singapore, 1995); A. Denner, Fortschr. Phys. 41, 307 (1993).

[48] S. Heinemeyer, W. Hollik, and G. Weiglein, Phys. Rep. 425, 265 (2006).

[49] T. Hahn, FEYNARTS 3.2, FORMCALC and LOOPTOOLS user's guides, available from http://www.feynarts.de; Comput. Phys. Commun. 168, 78 (2005).

[50] S. Heinemeyer, W. Hollik, and G. Weiglein, Comput. Phys. Commun. 124, 76 (2000); S. Heinemeyer and T. Hahn, http://www.feynhiggs.de/.

[51] F. Yuasa et al., Prog. Theor. Phys. Suppl. 138, 18 (2000); J. Fujimoto et al., Comput. Phys. Commun. 153, 106 (2003); Phys. Rev. D 75, 113002 (2007).

[52] N. Baro, F. Boudjema, and A. Semenov, Phys. Rev. D 78, 115003 (2008); N. Baro and F. Boudjema, Phys. Rev. D 80, 076010 (2009).

[53] A. Dabelstein, Z. Phys. C 67, 495 (1995); Nucl. Phys. B456, 25 (1995).

[54] M. Carena, S. Heinemeyer, C.E. M. Wagner, and G. Weiglein, Eur. Phys. J. C 26, 601 (2003).

[55] J.A. Grifols and A. Méndez, Phys. Rev. D 22, 1725 (1980).

[56] J. A. Grifols and J. Solà, Phys. Rev. D 23, 95 (1981).

[57] A. Arhrib, M. Capdequi Peyranere, W. Hollik, and G. Moultaka, Nucl. Phys. B581, 34 (2000).

[58] O. Brein and T. Hahn, Eur. Phys. J. C 52, 397 (2007).

[59] F. Mahmoudi and O. Stal, Phys. Rev. D 81, 035016 (2010).

[60] CDF and D0 Collaborations, arXiv:0903.4001; N. Krumnack, arXiv:0910.3353. 